\title{
Metrized Quantum Vector Bundles over Quantum Tori Built from Riemannian Metrics and Rosenberg's Levi-Civita Connections
}

\author{
Leonard HUANG
}

Department of Mathematics, University of Colorado at Boulder, Campus Box 395, 2300 Colorado Avenue, Boulder, CO 80309-0395, USA

E-mail: Leonard.Huang@Colorado.EDU

Received March 13, 2018, in final form July 21, 2018; Published online July 29, 2018

https://doi.org/10.3842/SIGMA.2018.079

\begin{abstract}
We build metrized quantum vector bundles, over a generically transcendental quantum torus, from Riemannian metrics, using Rosenberg's Levi-Civita connections for these metrics. We also prove that two metrized quantum vector bundles, corresponding to positive scalar multiples of a Riemannian metric, have distance zero between them with respect to the modular Gromov-Hausdorff propinquity.
\end{abstract}

Key words: quantum torus; generically transcendental; quantum metric space; metrized quantum vector bundle; Riemannian metric; Levi-Civita connection

2010 Mathematics Subject Classification: 46L08; 46L57; 46L87; 37A55; 58B34

\section{Introduction}

This paper was inspired by an apparent connection between Jonathan Rosenberg's work on Riemannian metrics on a generically transcendental quantum torus and Levi-Civita connections for these metrics [14], and Frédéric Latrémolière's work on metrized quantum vector bundles and the modular Gromov-Hausdorff propinquity [6].

The subject of the Gromov-Hausdorff propinquity has its origin in Marc Rieffel's observation [12] that in certain papers on theoretical physics, statements can be found regarding the convergence of a sequence of operator algebras to an operator algebra. He deduced that the bookkeeping device used by the authors of these papers to prove convergence can be described as a metric structure on unital $C^{*}$-algebras. Seeing that the Gromov-Hausdorff distance for compact metric spaces enables us to discuss the convergence of a sequence of compact metric spaces to a compact metric space, he defined for the class of order-unit spaces endowed with a special metric structure (called the compact quantum metric spaces) an analogous distance called the quantum Gromov-Hausdorff distance. The relation between order-unit spaces and $C^{*}$-algebras is made clear when one knows that the space of self-adjoint elements of a unital $C^{*}$-algebra is an order-unit space.

The quantum Gromov-Hausdorff distance suffers from some deficiencies. Designed for orderunit spaces, it does not incorporate the multiplicative structure of a $C^{*}$-algebra. Also, it was unknown if distance zero between two $C^{*}$-algebras necessarily means that they are $*$-isomorphic. These problems were settled when Latrémolière defined, in [4], the quantum Gromov-Hausdorff propinquity for the class of unital $C^{*}$-algebras endowed with a special metric structure (called the quantum compact metric spaces).

Recently, Latrémolière was able to generalize the Gromov-Hausdorff propinquity to Hilbert $C^{*}$-modules over a quantum compact metric space endowed with a special metric structure [6]. He calls these objects metrized quantum vector bundles, regarding them to be a noncommutative 
generalization of vector bundles over a compact Riemannian manifold endowed with a metric. The modular Gromov-Hausdorff propinquity then allows us to formalize the concept of convergence for metrized quantum vector bundles. Distance zero between metrized quantum vector bundles is equivalent to the existence of an isomorphism between them, in terms of their Hilbert$C^{*}$-module structures and their metric structures.

In Section 3, we will define Riemannian metrics on generically transcendental quantum tori and provide a brief overview of Rosenberg's work on Levi-Civita connections for these metrics.

In Section 4, we will define Latrémolière's quantum compact metric spaces and metrized quantum vector bundles. We will then show how to build a metrized quantum vector bundle from a Riemannian metric on a generically transcendental quantum torus and the Levi-Civita connection for the metric.

In Section 5, we will prove that two metrized quantum vector bundles, corresponding to positive scalar multiples of a Riemannian metric, have distance zero between them with respect to the modular Gromov-Hausdorff propinquity.

In Section 6, we will pose some open questions that serve as the basis for future work in this area.

\section{Preliminaries}

This section serves to standardize notation and conventions.

Let $\mathbb{N}$ denote the set of positive integers, and for each $m \in \mathbb{N}$, let $[m] \stackrel{\mathrm{df}}{=} \mathbb{N}_{\leq m}$.

Throughout this paper, fix $n \in \mathbb{N}$ as well as a generically transcendental ${ }^{1}$ skew-adjoint $(n \times n)$-matrix $\Theta$ having entries in $\mathbb{C}$.

Fix also an arbitrary norm $N$ on $\mathbb{R}^{n}$. Of particular physical importance is the Euclidean norm on $\mathbb{R}^{n}$.

Let $A_{\Theta}$ denote the $n$-dimensional quantum torus corresponding to $\Theta$, which is the universal $C^{*}$-algebra generated by $n$ unitary elements $u_{1}, \ldots, u_{n}$ satisfying the relation $u_{k} u_{j}=e^{2 \pi i \Theta_{j k}} u_{j} u_{k}$ for all $j, k \in[n]$.

Let $\alpha$ denote the canonical action of $\mathbb{T}^{n}$ on $A_{\Theta}$ given by $\alpha_{\boldsymbol{t}}\left(u_{j}\right)=t_{j} \cdot u_{j}$ for all $\boldsymbol{t} \in \mathbb{T}^{n}$ and $j \in[n]$, where $\boldsymbol{t}=\left(t_{j}\right)_{j \in[n]}$. Then let $\partial_{1}, \ldots, \partial_{n}$ denote the coordinate directional-derivative operators associated to $\alpha$.

Let $A_{\Theta}^{\infty}$ denote the *-subalgebra of smooth elements of $A_{\Theta}$ for $\alpha$, which we refer to as the $n$-dimensional smooth quantum torus corresponding to $\Theta$. Then let $\mathcal{D}_{\Theta}$ denote the $\mathbb{R}$-vector space of $*$-derivations on $A_{\Theta}^{\infty}$.

Given a vector space $V$, a seminorm $L$ on $V$, and $r \in \mathbb{R}_{>0}$, let $L^{r} \stackrel{\text { df }}{=}\{v \in V \mid L(v) \leq r\}$.

Given a $*$-algebra $A$, let its set of self-adjoint elements and its set of skew-adjoint elements be denoted by $A_{\text {sa }}$ and $A_{\text {ska }}$ respectively, and the $*$-algebra of $(n \times n)$-matrices with entries in $A$ be denoted by $M_{n}(A)$.

Given a $C^{*}$-algebra $A$, let its state space be denoted by $\mathscr{S}(A)$.

\section{Generically transcendental quantum tori as noncommutative Riemannian manifolds}

This section gives a brief overview of Riemannian metrics on a generically transcendental quantum torus and Rosenberg's Levi-Civita connections for these metrics, as defined in [14].

Throughout this section, $A$ denotes a unital $C^{*}$-algebra.

\footnotetext{
${ }^{1}$ This is a rather complicated Diophantine condition that is defined in [2].
} 
Definition 3.1. Let $\chi(A)$ denote the free left $A$-module with rank $n$ whose underlying $\mathbb{C}$-vector space is $A^{n}$, where the left action $\bullet$ of $a \in A$ on $X \in \chi(A)$ is given by left multiplication of each component of $X$ by $a$. For every $j \in[n]$, let $\mathbf{e}_{j}$ denote the element of $\chi(A)$ that has $1_{A}$ in the $j$-th component and $0_{A}$ 's elsewhere. Define the standard $A$-valued inner product $\langle\cdot \mid \cdot\rangle_{\mathrm{st}}$ and its associated (standard) norm $\|\cdot\|_{\text {st }}$ on $\chi(A)$ by

$$
\begin{aligned}
\forall a_{1}, \ldots, a_{n}, b_{1}, \ldots, b_{n} \in A: \quad & \left\langle\sum_{j=1}^{n} a_{j} \bullet \mathbf{e}_{j} \mid \sum_{j=1}^{n} b_{j} \bullet \mathbf{e}_{j}\right\rangle \stackrel{\mathrm{dt}}{=} \sum_{j=1}^{n} a_{j} b_{j}^{*}, \\
& \left\|\sum_{j=1}^{n} a_{j} \bullet \mathbf{e}_{j}\right\|_{\mathrm{st}} \stackrel{\mathrm{df}}{=}\left\|\sum_{j=1}^{n} a_{j} a_{j}^{*}\right\|_{A}^{\frac{1}{2}} \cdot
\end{aligned}
$$

Then $\left(\chi(A),\langle\cdot \mid \cdot\rangle_{\text {st }}\right)$ is a left Hilbert $A$-module.

Let $\mathbb{L}\left(\chi(A),\langle\cdot \mid \cdot\rangle_{\mathrm{st}}\right)$ denote the $C^{*}$-algebra of adjointable maps on $\left(\chi(A),\langle\cdot \mid \cdot\rangle_{\mathrm{st}}\right)$. Define a unital algebraic $*$-anti-isomorphism $T: M_{n}(A) \rightarrow \mathbb{L}\left(\chi(A),\langle\cdot \mid \cdot\rangle_{\mathrm{st}}\right)$ by

$$
\forall a_{1}, \ldots, a_{n} \in A: \quad T_{g}\left(\sum_{j=1}^{n} a_{j} \bullet \mathbf{e}_{j}\right) \stackrel{\mathrm{df}}{=} \sum_{k=1}^{n}\left(\sum_{j=1}^{n} a_{j} g_{j k}\right) \bullet \mathbf{e}_{k} .
$$

We can define a $C^{*}$-algebraic norm $\|\cdot\|_{M_{n}(A)}$ on $M_{n}(A)$ by $\|g\|_{M_{n}(A)} \stackrel{\mathrm{df}}{=}\left\|T_{g}\right\|_{\mathbb{L}\left(\chi(A),\langle\cdot \mid \cdot\rangle_{\mathrm{st}}\right)}$ for all $g \in M_{n}(A)$. Henceforth, we will view $M_{n}(A)$ as a $C^{*}$-algebra.

Proposition 3.2. Let $g \in M_{n}(A)$ be positive and invertible. Define a map $\langle\cdot \mid \cdot\rangle_{g}: \chi(A) \times \chi(A) \rightarrow A$ by

$$
\forall X, Y \in \chi(A): \quad\langle X \mid Y\rangle_{g} \stackrel{\mathrm{df}}{=}\left\langle T_{g}(X) \mid Y\right\rangle_{\mathrm{st}} .
$$

Then the following statements hold:

1) $\left(\chi(A),\langle\cdot \mid \cdot\rangle_{g}\right)$ is a left Hilbert $A$-module (we denote the associated norm on $\chi(A)$ by $\left.\|\cdot\|_{g}\right)$,

2) $\|X\|_{g}=\left\|T_{\sqrt{g}}(X)\right\|_{\text {st }}$ for all $X \in \chi(A)$,

3) $\left\langle\mathbf{e}_{j} \mid \mathbf{e}_{k}\right\rangle_{g}=g_{j k}$ for all $j, k \in[n]$.

Proof. By hypothesis, $g$ has an invertible positive square root in $M_{n}(A)$, so

$$
\forall X, Y \in \chi(A): \quad\langle X \mid Y\rangle_{g}=\left\langle T_{g}(X) \mid Y\right\rangle_{\mathrm{st}}=\left\langle T_{\sqrt{g}}(X) \mid T_{\sqrt{g}}^{*}(Y)\right\rangle_{\mathrm{st}}=\left\langle T_{\sqrt{g}}(X) \mid T_{\sqrt{g}}(Y)\right\rangle_{\mathrm{st}} .
$$

We can thus see that $\langle\cdot \mid \cdot\rangle_{g}$ is a sesquilinear form. Furthermore,

$$
\forall X, Y \in \chi(A): \quad\langle Y \mid X\rangle_{g}=\left\langle T_{\sqrt{g}}(Y) \mid T_{\sqrt{g}}(X)\right\rangle_{\mathrm{st}}=\left\langle T_{\sqrt{g}}(X) \mid T_{\sqrt{g}}(Y)\right\rangle_{\mathrm{st}}^{*}=\langle X \mid Y\rangle_{g}^{*} .
$$

As $T_{\sqrt{g}}$ is $A$-linear, we find that

$$
\begin{aligned}
\forall a \in A, \forall X, Y \in \chi(A): \quad\langle a \bullet X \mid Y\rangle_{g} & =\left\langle T_{\sqrt{g}}(a \bullet X) \mid T_{\sqrt{g}}(Y)\right\rangle_{\mathrm{st}} \\
& =\left\langle a \bullet T_{\sqrt{g}}(X) \mid T_{\sqrt{g}}(Y)\right\rangle_{\mathrm{st}} \\
& =a\left\langle T_{\sqrt{g}}(X) \mid T_{\sqrt{g}}(Y)\right\rangle_{\mathrm{st}} \\
& =a\langle X \mid Y\rangle_{g} .
\end{aligned}
$$


Next, we have for all $X \in \chi(A)$ that

$$
\langle X \mid X\rangle_{g}=\left\langle T_{\sqrt{g}}(X) \mid T_{\sqrt{g}}(X)\right\rangle_{\mathrm{st}} \geq_{A} 0_{A},
$$

and if $\langle X \mid X\rangle_{g}=0_{A}$, then $\left\langle T_{\sqrt{g}}(X) \mid T_{\sqrt{g}}(X)\right\rangle_{\text {st }}=0_{A}$, so $T_{\sqrt{g}}(X)=0_{\chi(A)}$ and thus $X=0_{\chi(A)}$. Continuing,

$$
\forall X \in \chi(A): \quad\|X\|_{g}=\left\|\langle X \mid X\rangle_{g}\right\|_{A}^{\frac{1}{2}}=\left\|\left.\left\langle T_{\sqrt{g}}(X) \mid T_{\sqrt{g}}(X)\right\rangle_{\mathrm{st}}\right|_{A} ^{\frac{1}{2}}=\right\| T_{\sqrt{g}}(X) \|_{\mathrm{st}},
$$

so if $\left(X_{k}\right)_{k \in \mathbb{N}}$ is a Cauchy sequence in $\chi(A)$ with respect to $\|\cdot\|_{g}$, then $\left(T_{\sqrt{g}}\left(X_{k}\right)\right)_{k \in \mathbb{N}}$ is a Cauchy sequence in $\chi(A)$ with respect to $\|\cdot\|_{\text {st }}$ that has a $\|\cdot\|_{\text {st }}$-limit $X^{\prime}$, as $\left(\chi(A),\|\cdot\|_{\text {st }}\right)$ is a complete metric space. Hence,

$$
\lim _{k \rightarrow \infty}\left\|X_{k}-T_{\sqrt{g}}^{-1}\left(X^{\prime}\right)\right\|_{g}=\lim _{k \rightarrow \infty}\left\|T_{\sqrt{g}}\left(X_{k}-T_{\sqrt{g}}^{-1}\left(X^{\prime}\right)\right)\right\|_{\mathrm{st}}=\lim _{k \rightarrow \infty}\left\|T_{\sqrt{g}}\left(X_{k}\right)-X^{\prime}\right\|_{\mathrm{st}}=0 .
$$

Therefore, $\left(\chi(A),\|\cdot\|_{g}\right)$ is a complete metric space, which establishes (1) and (2).

Finally, (3) follows from the definition of $\langle\cdot \mid \cdot\rangle_{g}$.

The following lemma will be needed in Section 4 .

Lemma 3.3. Let $g, h \in M_{n}(A)$ be positive and invertible. Then

$$
\frac{1}{\left\|\sqrt{h} \sqrt{g^{-1}}\right\|_{M_{n}(A)}}\|\cdot\|_{h} \leq\|\cdot\|_{g} \leq\left\|\sqrt{g} \sqrt{h^{-1}}\right\|_{M_{n}(A)}\|\cdot\|_{h},
$$

so $\|\cdot\|_{g}$ and $\|\cdot\|_{h}$ are equivalent norms on $\chi(A)$. In particular,

$$
\frac{1}{\|\sqrt{h}\|_{M_{n}(A)}}\|\cdot\|_{h} \leq\|\cdot\|_{\mathrm{st}} \leq\left\|\sqrt{h^{-1}}\right\|_{M_{n}(A)}\|\cdot\|_{h}
$$

Proof. Observe that

$$
\begin{aligned}
\forall X \in \chi(A): \quad\|X\|_{h} & =\left\|T_{\sqrt{h}}(X)\right\|_{\mathrm{st}} \quad(\text { by }(2) \text { of Proposition 3.2) } \\
& =\left\|T_{\sqrt{h}} T_{\sqrt{g}}^{-1} T_{\sqrt{g}}(X)\right\|_{\mathrm{st}} \\
& =\left\|T_{\sqrt{h} \sqrt{g^{-1}}} T_{\sqrt{g}}(X)\right\|_{\mathrm{st}} \\
& \leq\left\|T_{\sqrt{h} \sqrt{g^{-1}}}\right\|_{\mathbb{L}\left(\chi(A),\langle\cdot \cdot\rangle_{\mathrm{st}}\right)}\left\|T_{\sqrt{g}}(X)\right\|_{\mathrm{st}} \\
& =\left\|T_{\sqrt{h} \sqrt{g^{-1}}}\right\|_{\mathbb{L}\left(\chi(A),\langle\cdot \cdot \cdot\rangle_{\mathrm{st}}\right.}\|X\|_{g} \quad \text { (by (2) of Proposition 3.2 again) } \\
& =\left\|\sqrt{h} \sqrt{g^{-1}}\right\|_{M_{n}(A)}\|X\|_{g} .
\end{aligned}
$$

Interchanging $g$ and $h$ in the relations above, we get $\|X\|_{g} \leq\left\|\sqrt{h} \sqrt{g^{-1}}\right\|_{M_{n}(A)}\|X\|_{h}$ for all $X \in \chi(A)$, so

$$
\frac{1}{\left\|\sqrt{h} \sqrt{g^{-1}}\right\|_{M_{n}(A)}}\|\cdot\|_{h} \leq\|\cdot\|_{g} \leq\left\|\sqrt{g} \sqrt{h^{-1}}\right\|_{M_{n}(A)}\|\cdot\|_{h}
$$

as required.

Finally, as $\|\cdot\|_{\text {st }}=\|\cdot\|_{\mathrm{I}}$, where $\mathrm{I}$ is the identity of $M_{n}(A)$, the second part comes from letting $g=\mathrm{I}$. 
It was established in [2] that

$$
\mathcal{D}_{\Theta}=\left\{\sum_{j=1}^{n} r_{j} \cdot \partial_{j}+\operatorname{ad}_{a} \mid r_{1}, \ldots, r_{n} \in \mathbb{R} \text { and } a \in\left(A_{\Theta}^{\infty}\right)_{\text {ska }}\right\},
$$

where the hypothesis that $\Theta$ is generically transcendental plays a role. For any $a, b \in A_{\Theta}$, we have $\operatorname{ad}_{a}=\operatorname{ad}_{b}$ if and only if $a-b \in \mathbb{C} \cdot 1_{A_{\Theta}}$, so if $\tau$ denotes the faithful tracial state on $A_{\Theta}$, then (3.1) can be rewritten as

$$
\mathcal{D}_{\Theta}=\left\{\sum_{j=1}^{n} r_{j} \cdot \partial_{j}+\operatorname{ad}_{a-\tau(a) \cdot 1_{A_{\Theta}}} \mid r_{1}, \ldots, r_{n} \in \mathbb{R} \text { and } a \in\left(A_{\Theta}^{\infty}\right)_{\text {ska }}\right\} .
$$

Now, for every $a \in\left(A_{\Theta}\right)_{\text {ska }}$, the following observations can be made:

- as $\tau\left(1_{A_{\Theta}}\right)=1$, we have $\tau\left(a-\tau(a) \cdot 1_{A_{\Theta}}\right)=0$,

- as $a$ is skew-adjoint, we have $a=i b$ for some self-adjoint $b \in A_{\Theta}$, so

$$
\left[a-\tau(a) \cdot 1_{A_{\Theta}}\right]^{*}=a^{*}-\overline{\tau(a)} \cdot 1_{A_{\Theta}} .
$$

Hence, $a-\tau(a) \cdot 1_{A_{\Theta}}$ is skew-adjoint as well.

By [14, Theorem 1.4], the decomposition of every element of $\mathcal{D}_{\Theta}$ into an $\mathbb{R}$-linear combination of the $\partial_{j}$ 's and an inner $*$-derivation is unique, so the observations above tell us that $\mathcal{D}_{\Theta}$ can be linearly parametrized by the normed $\mathbb{R}$-vector space $\mathbb{R}^{n} \times\left[\left(A_{\Theta}^{\infty}\right)_{\text {ska }} \cap \operatorname{ker}(\tau)\right]$, whose norm $\|\cdot\|$ we have chosen to be defined by

$$
\forall r_{1}, \ldots, r_{n} \in \mathbb{R}, \forall a \in\left(A_{\Theta}^{\infty}\right)_{\mathrm{ska}} \cap \operatorname{ker}(\tau): \quad\left\|\left(r_{1}, \ldots, r_{n} ; a\right)\right\| \stackrel{\mathrm{df}}{=} N\left(r_{1}, \ldots, r_{n}\right)+\|a\|_{A_{\Theta}} .
$$

We may then transfer $\|\cdot\|$ to an $\mathbb{R}$-vector-space norm $\|\cdot\|_{\mathcal{D}_{\Theta}}$ on $\mathcal{D}_{\Theta}$ in an obvious way.

For the rest of this paper, we will let $\chi_{\Theta} \stackrel{\mathrm{df}}{=} \chi\left(A_{\Theta}\right)$ and

$$
\chi_{\Theta}^{\infty} \stackrel{\mathrm{df}}{=}\left\{\sum_{j=1}^{n} a_{j} \bullet \mathbf{e}_{j} \in \chi_{\Theta} \mid a_{1}, \ldots, a_{n} \in A_{\Theta}^{\infty}\right\} .
$$

Definition 3.4 ([14]). A Riemannian metric is an element of $M_{n}\left(A_{\Theta}\right)$ that is positive, is invertible, and has all entries in $\left(A_{\Theta}^{\infty}\right)_{\mathrm{sa}}$.

Definition $3.5([14])$. A connection for a Riemannian metric $g$ is a map $\nabla: \mathcal{D}_{\Theta} \times \chi_{\Theta}^{\infty} \rightarrow \chi_{\Theta}^{\infty}$ satisfying the following four properties:

1) $\nabla$ is $\mathbb{R}$-linear in the first variable and $\mathbb{C}$-linear in the second,

2) $\nabla_{\delta}(a \bullet X)=\delta(a) \bullet X+a \bullet \nabla_{\delta}(X)$ for all $\delta \in \mathcal{D}_{\Theta}, a \in A_{\Theta}^{\infty}$ and $X \in \chi_{\Theta}^{\infty}$ (this property is known as the Leibniz rule),

3) $\nabla_{\mathrm{ad}_{a}}(X)=a \bullet X$ for all $a \in\left(A_{\Theta}^{\infty}\right)_{\text {ska }} \cap \operatorname{ker}(\tau)$ and $X \in \chi_{\Theta}^{\infty}$,

4) $\left\langle\nabla_{\partial_{j}}\left(\mathbf{e}_{k}\right) \mid \mathbf{e}_{l}\right\rangle_{g}$ is self-adjoint for all $j, k, l \in[n]$.

If $\nabla$ further satisfies the following two properties, then we call it a Levi-Civita connection for $g$ :

5) $\nabla_{\partial_{j}}\left(\mathbf{e}_{k}\right)=\nabla_{\partial_{k}}\left(\mathbf{e}_{j}\right)$ for all $j, k \in[n]$ (this property is known as the torsion-freeness of $\nabla$ (we will not need this property in our proofs), 
6) $\delta\left(\langle X \mid Y\rangle_{g}\right)=\left\langle\nabla_{\delta}(X) \mid Y\right\rangle_{g}+\left\langle X \mid \nabla_{\delta}(Y)\right\rangle_{g}$ for all $\delta \in \mathcal{D}_{\Theta}$ and $X, Y \in \chi_{\Theta}^{\infty}$ (this property is known as the compatibility of $\nabla$ with $g)$.

We now state, for the class of all generically transcendental quantum tori, a noncommutative version of the fundamental theorem of Riemannian geometry.

Theorem 3.6 ([14]). For every Riemannian metric g, there exists a unique Levi-Civita connection $\nabla^{g}$ for $g$, which necessarily satisfies the following identity ${ }^{2}$ :

$$
\forall j, k, l \in[n]: \quad\left\langle\nabla_{\partial_{j}}^{g}\left(\mathbf{e}_{k}\right) \mid \mathbf{e}_{l}\right\rangle_{g}=g_{j k l}^{\natural} \stackrel{\mathrm{df}}{=} \frac{1}{2} \cdot\left[\partial_{j}\left(g_{k l}\right)+\partial_{k}\left(g_{j l}\right)-\partial_{l}\left(g_{j k}\right)\right] .
$$

\section{Metrized quantum vector bundles over generically transcendental quantum tori}

We will first define quantum compact metric spaces and metrized quantum vector bundles. We will then show how to build metrized quantum vector bundles over a generically transcendental quantum torus from Riemannian metrics, using Rosenberg's Levi-Civita connections for these metrics.

Definition 4.1 ([6]). An admissible function is a function $F:[0, \infty)^{4} \rightarrow[0, \infty)$ with the following properties:

- $F$ is non-decreasing in each argument,

- $w z+x y \leq F(w, x, y, z)$ for all $w, x, y, z \in[0, \infty)$.

If $F(w, x, y, z)=w z+x y$ for all $w, x, y, z \in[0, \infty)$, then we say that it is Leibniz.

Definition 4.2 ([5, 6]). Let $F$ be an admissible function. An $F$-quasi-Leibniz quantum compact metric space is then an ordered pair $(A, \mathrm{~L})$ satisfying the following six properties:

1) $A$ is a unital $C^{*}$-algebra,

2) $\mathrm{L}$ is a seminorm defined on a dense Jordan-Lie subalgebra of $A_{\mathrm{sa}}$,

3) $\{a \in \operatorname{Dom}(\mathrm{L}) \mid \mathrm{L}(a)=0\}=\mathbb{R} \cdot 1_{A}$,

4) the Monge-Kantorovich metric of $\mathrm{L}$, which is the function $\mathrm{mk}_{\mathrm{L}}: \mathscr{S}(A) \times \mathscr{S}(A) \rightarrow[0, \infty]$ defined by

$$
\forall \phi, \psi \in \mathscr{S}(A): \quad \mathrm{mk}_{\mathrm{L}}(\phi, \psi) \stackrel{\mathrm{df}}{=} \sup \left(\left\{\mid \phi(a)-\psi(a) \| a \in \mathrm{L}^{1}\right\}\right),
$$

metrizes the weak-* topology on $\mathscr{S}(A)$,

5) $\mathrm{L}^{1}$ is $\|\cdot\|_{A^{-}}$-closed in $A$ (equivalently, $\mathrm{L}^{r}$ is $\|\cdot\|_{A^{-}}$-losed in $A$ for every $r \in \mathbb{R}_{>0}$ ),

6) for all $a, b \in \operatorname{Dom}(\mathrm{L})$, we have

$$
\max \left(\mathrm{L}\left(\frac{1}{2} \cdot(a b+b a)\right), \mathrm{L}\left(\frac{1}{2 i} \cdot(a b-b a)\right)\right) \leq F\left(\|a\|_{A},\|b\|_{A}, \mathrm{~L}(a), \mathrm{L}(b)\right) .
$$

If $F$ is implicitly understood, then we simply call $(A, \mathrm{~L})$ a quasi-Leibniz quantum compact metric space, and if $F$ is Leibniz, then we simply call $(A, \mathrm{~L})$ a Leibniz quantum compact metric space. The seminorm $\mathrm{L}$ is called a Lipschitz seminorm, and it is what endows $A$ with its special metric structure.

\footnotetext{
${ }^{2}$ This identity is used to prove the classical version of the fundamental theorem of Riemannian geometry.
} 
Example 4.3. The following classes of $C^{*}$-algebras can be endowed with Lipschitz seminorms that turn them into quasi-Leibniz quantum compact metric spaces: $\mathrm{AF} C^{*}$-algebras [1], curved noncommutative tori [3], noncommutative solenoids [8], and $C^{*}$-algebras equipped with an ergodic action of a compact group [10].

Definition $4.4([6])$. An admissible triple is an ordered triple $(F, G, H)$ with the following properties:

- $F$ is an admissible function,

- $G:[0, \infty)^{3} \rightarrow[0, \infty)$ and $H:[0, \infty)^{2} \rightarrow[0, \infty)$,

- $G$ and $H$ are non-decreasing in each of their arguments,

- $(x+y) z \leq G(x, y, z)$ and $2 x y \leq H(x, y)$ for all $x, y, z \in[0, \infty)$.

Definition 4.5 ([6]). Let $(F, G, H)$ be an admissible triple. An $(F, G, H)$-metrized quantum vector bundle is then an ordered 5 -tuple $(\mathrm{X},\langle\cdot \mid \cdot\rangle, \mathrm{D}, A, \mathrm{~L})$ satisfying the following seven properties:

1) $(A, \mathrm{~L})$ is an $F$-quasi-Leibniz quantum compact metric space,

2) $(\mathrm{X},\langle\cdot \mid \cdot\rangle)$ is a left Hilbert $A$-module (we denote the associated norm on $\mathrm{X}$ by $\|\cdot\|_{\langle\cdot \mid \cdot\rangle}$ ),

3) $\mathrm{D}$ is a norm (not merely a seminorm) defined on a $\|\cdot\|_{\langle\cdot \mid \cdot\rangle}$-dense $\mathbb{C}$-linear subspace of $X$,

4) $\|\zeta\|_{\langle\cdot \mid \cdot\rangle} \leq \mathrm{D}(\zeta)$ for all $\zeta \in \operatorname{Dom}(\mathrm{D})$,

5) $\mathrm{D}^{1}$ is $\|\cdot\|_{\langle\cdot \mid \cdot\rangle}$-compact in $\mathrm{X}$ (equivalently, $\mathrm{D}^{r}$ is $\|\cdot\|_{\langle\cdot \mid \cdot\rangle}$-compact in $\mathrm{X}$ for every $r \in \mathbb{R}_{>0}$ ),

6) for all $a \in \operatorname{Dom}(\mathrm{L})$ and $\zeta \in \operatorname{Dom}(\mathrm{D})$, we have $a \bullet \zeta \in \operatorname{Dom}(\mathrm{D})$ and

$$
\mathrm{D}(a \bullet \zeta) \leq G\left(\|a\|_{A}, \mathrm{~L}(a), \mathrm{D}(\zeta)\right),
$$

7) for all $\zeta, \eta \in \operatorname{Dom}(\mathrm{D})$, we have $\Re(\langle\zeta \mid \eta\rangle), \Im(\langle\zeta \mid \eta\rangle) \in \operatorname{Dom}(\mathrm{L})$ and

$$
\max (\mathrm{L}(\Re(\langle\zeta \mid \eta\rangle)), \mathrm{L}(\Im(\langle\zeta \mid \eta\rangle))) \leq H(\mathrm{D}(\zeta), \mathrm{D}(\eta)) .
$$

If $(F, G, H)$ is implicitly understood, then we simply call $(\mathrm{X},\langle\cdot \mid \cdot\rangle, \mathrm{D}, A, \mathrm{~L})$ a metrized quantum vector bundle.

Example 4.6. In [6], Latrémolière constructed metrized quantum vector bundles from actual vector bundles over compact Riemannian manifolds that provide motivating examples for Definition 4.5. In the same paper, he also showed that free Hilbert modules over the underlying unital $C^{*}$-algebra of a quasi-Leibniz quantum compact metric space can be turned into metrized quantum vector bundles. The tools developed in [6] are then applied in [7] to prove the convergence of Heisenberg modules over quantum 2-tori with respect to the modular Gromov-Hausdorff propinquity.

Viewing $\mathbb{T}^{n}$ as $\mathbb{R}^{n} / \mathbb{Z}^{n}$, we may define an $N$-dependent continuous length function $\ell$ on $\mathbb{T}^{n}$ by

$$
\forall s \in\left[-\frac{1}{2}, \frac{1}{2}\right)^{n}: \quad \ell\left(s+\mathbb{Z}^{n}\right) \stackrel{\text { df }}{=} N(s) .
$$

Using $\ell$, we may then define an $N$-dependent seminorm $\mathrm{L}$ on a dense Jordan-Lie subalgebra of $\left(A_{\Theta}\right)_{\mathrm{sa}}$ by

$$
\begin{aligned}
& \operatorname{Dom}(\mathrm{L})=\left\{a \in\left(A_{\Theta}\right)_{\mathrm{sa}} \mid \sup \left(\left\{\frac{\left\|\alpha_{\boldsymbol{t}}(a)-a\right\|_{A_{\Theta}}}{\ell(\boldsymbol{t})} \mid \boldsymbol{t} \in \mathbb{T}^{n} \backslash\{\mathbf{0}\}\right\}\right)<\infty\right\}, \\
& \forall a \in \operatorname{Dom}(\mathrm{L}): \quad \mathrm{L}(a) \stackrel{\mathrm{df}}{=} \sup \left(\left\{\frac{\left\|\alpha_{\boldsymbol{t}}(a)-a\right\|_{A_{\Theta}}}{\ell(\boldsymbol{t})} \mid \boldsymbol{t} \in \mathbb{T}^{n} \backslash\{\mathbf{0}\}\right\}\right) .
\end{aligned}
$$


The action $\alpha$ of $\mathbb{T}^{n}$ on $A_{\Theta}$ is ergodic, so $\left(A_{\Theta}, \mathrm{L}\right)$ is a Leibniz quantum compact metric space by [10, Section 2]. Furthermore, according to [12, Proposition 8.6], we have

$$
\begin{aligned}
& \forall a \in\left(A_{\Theta}^{\infty}\right)_{\mathrm{sa}}: \\
& \mathrm{L}(a)=\max \left(\left\{\left.\left\|\sum_{j=1}^{n} r_{j} \cdot \partial_{j}(a)\right\|\right|_{A_{\Theta}} \mid r_{1}, \ldots, r_{n} \in \mathbb{R} \text { and } N\left(r_{1}, \ldots, r_{n}\right) \leq 1\right\}\right) .
\end{aligned}
$$

This alternative expression for $\mathrm{L}$ on $\left(A_{\Theta}^{\infty}\right)_{\text {sa }}$ will play an important role in what is to follow.

Lemma 4.7. Let $\delta \in \mathcal{D}_{\Theta}$. Then $\|\delta(a)\|_{A_{\Theta}} \leq\left[2\|a\|_{A_{\Theta}}+\mathrm{L}(a)\right]\|\delta\|_{\mathcal{D}_{\Theta}}$ for all $a \in\left(A_{\Theta}^{\infty}\right)_{\mathrm{sa}}$.

Proof. Write $\delta=\sum_{j=1}^{n} r_{j} \cdot \partial_{j}+\operatorname{ad}_{b}$, where $r_{1}, \ldots, r_{n} \in \mathbb{R}$ and $b \in\left(A_{\Theta}^{\infty}\right)_{\text {ska }} \cap \operatorname{ker}(\tau)$. As

$$
\|\delta\|_{\mathcal{D}_{\Theta}}=N\left(r_{1}, \ldots, r_{n}\right)+\|b\|_{A_{\Theta}}
$$

we have $N\left(r_{1}, \ldots, r_{n}\right) \leq\|\delta\|_{\mathcal{D}_{\Theta}}$ and $\|b\|_{A_{\Theta}} \leq\|\delta\|_{\mathcal{D}_{\Theta}}$. Hence,

$$
\begin{aligned}
\|\delta(a)\|_{A_{\Theta}} & =\left\|\sum_{j=1}^{n} r_{j} \cdot \partial_{j}(a)+[b, a]\right\|_{A_{\Theta}} \\
& \leq\left\|\sum_{j=1}^{n} r_{j} \cdot \partial_{j}(a)\right\|_{A_{\Theta}}+\|[b, a]\|_{A_{\Theta}} \\
& \leq\left\|\sum_{j=1}^{n} r_{j} \cdot \partial_{j}(a)\right\|_{A_{\Theta}}+2\|b\|_{A_{\Theta}}\|a\|_{A_{\Theta}} \\
& \leq\|\delta\|_{\mathcal{D}_{\Theta}} \mathrm{L}(a)+2\|\delta\|_{\mathcal{D}_{\Theta}}\|a\|_{A_{\Theta}} \quad(\text { by }(4.1)) \\
& =\left[2\|a\|_{A_{\Theta}}+\mathrm{L}(a)\right]\|\delta\|_{\mathcal{D}_{\Theta}} \cdot
\end{aligned}
$$

This completes the proof.

Definition 4.8. For every Riemannian metric $g$, define an $N$-dependent seminorm $\|\cdot\| \|_{g}$ on $\chi_{\Theta}^{\infty}$ by

$$
\forall X \in \chi_{\Theta}^{\infty}: \quad\|X\|_{g} \stackrel{\mathrm{df}}{=} \sup \left(\left\{\left\|\nabla_{\delta}^{g}(X)\right\|_{g} \mid \delta \in \mathcal{D}_{\Theta} \text { and }\|\delta\|_{\mathcal{D}_{\Theta}} \leq 1\right\}\right),
$$

and an $N$-dependent norm $\mathrm{D}_{g}$ on $\chi_{\Theta}^{\infty}$ by $\mathrm{D}_{g} \stackrel{\mathrm{df}}{=} \max \left(\|\cdot\|_{g},\|\cdot\| \|_{g}\right) \cdot{ }^{3}$

Note: For the rest of this section only, $g$ denotes a Riemannian metric.

Proposition 4.9. Let $a \in\left(A_{\Theta}^{\infty}\right)_{\mathrm{sa}}$ and $X \in \chi_{\Theta}^{\infty}$. Then $a \bullet X \in \chi_{\Theta}^{\infty}$ and

$$
\mathrm{D}_{g}(a \bullet X) \leq G\left(\|a\|_{A_{\Theta}}, \mathrm{L}(a), \mathrm{D}_{g}(X)\right),
$$

where $G:[0, \infty)^{3} \rightarrow[0, \infty)$ is defined by $G(x, y, z) \stackrel{\text { df }}{=}(3 x+y) z$ for all $x, y, z \in[0, \infty)$.

\footnotetext{
${ }^{3}$ This is the point where Rosenberg's Levi-Civita connections come in.
} 
Proof. It is clear from the definition of $\chi_{\Theta}^{\infty}$ that $a \bullet X \in \chi_{\Theta}^{\infty}$.

Next, by the Leibniz rule,

$$
\forall \delta \in \mathcal{D}_{\Theta}: \quad \nabla_{\delta}^{g}(a \bullet X)=\delta(a) \bullet X+a \bullet \nabla_{\delta}^{g}(X) .
$$

Hence, we have for all $\delta \in \mathcal{D}_{\Theta}$ satisfying $\|\delta\|_{\mathcal{D}_{\Theta}} \leq 1$ that

$$
\begin{aligned}
\left\|\nabla_{\delta}^{g}(a \bullet X)\right\|_{g} & \leq\|\delta(a) \bullet X\|_{g}+\left\|a \bullet \nabla_{\delta}^{g}(X)\right\|_{g} \\
& \leq\|\delta(a)\|_{A_{\Theta}}\|X\|_{g}+\|a\|_{A_{\Theta}}\left\|\nabla_{\delta}^{g}(X)\right\|_{g} \\
& \leq\left[2\|a\|_{A_{\Theta}}+\mathrm{L}(a)\right]\|X\|_{g}+\|a\|_{A_{\Theta}}\left\|\nabla_{\delta}^{g}(X)\right\|_{g} \quad \text { (by Lemma 4.7) } \\
& \leq\left[2\|a\|_{A_{\Theta}}+\mathrm{L}(a)\right]\|X\|_{g}+\|a\|_{A_{\Theta}}\|X\|_{g}\|\delta\|_{\mathcal{D}_{\Theta}} \\
& \leq\left[2\|a\|_{A_{\Theta}}+\mathrm{L}(a)\right]\|X\|_{g}+\|a\|_{A_{\Theta}}\|X\|_{g} \\
& \leq\left[2\|a\|_{A_{\Theta}}+\mathrm{L}(a)\right] \mathrm{D}_{g}(X)+\|a\|_{A_{\Theta}} \mathrm{D}_{g}(X) \\
& =\left[3\|a\|_{A_{\Theta}}+\mathrm{L}(a)\right] \mathrm{D}_{g}(X),
\end{aligned}
$$

which immediately yields

$$
\|a \bullet X\|_{g} \leq\left[3\|a\|_{A_{\Theta}}+\mathrm{L}(a)\right] \mathrm{D}_{g}(X) .
$$

At the same time, it is straightforward to see that

$$
\|a \bullet X\|_{g} \leq\|a\|_{A_{\Theta}}\|X\|_{g} \leq\|a\|_{A_{\Theta}} \mathrm{D}_{g}(X) \leq\left[3\|a\|_{A_{\Theta}}+\mathrm{L}(a)\right] \mathrm{D}_{g}(X) .
$$

Therefore,

$$
\mathrm{D}_{g}(a \bullet X)=\max \left(\|a \bullet X\|_{g},\|a \bullet X\|_{g}\right) \leq G\left(\|a\|_{A_{\Theta}}, \mathrm{L}(a), \mathrm{D}_{g}(X)\right)
$$

as required.

Proposition 4.10. Let $X, Y \in \chi_{\Theta}^{\infty}$. Then $\Re\left(\langle X \mid Y\rangle_{g}\right), \Im\left(\langle X \mid Y\rangle_{g}\right) \in\left(A_{\Theta}^{\infty}\right)_{\mathrm{sa}}$ and

$$
\max \left(\mathrm{L}\left(\Re\left(\langle X \mid Y\rangle_{g}\right)\right), \mathrm{L}\left(\Im\left(\langle X \mid Y\rangle_{g}\right)\right)\right) \leq H\left(\mathrm{D}_{g}(X), \mathrm{D}_{g}(Y)\right),
$$

where $H:[0, \infty)^{2} \rightarrow[0, \infty)$ is defined by $H(x, y) \stackrel{\mathrm{df}}{=} 2 x y$ for all $x, y \in[0, \infty)$.

Proof. It is clear from the definition of $\chi_{\Theta}^{\infty}$ that $\Re\left(\langle X \mid Y\rangle_{g}\right), \Im\left(\langle X \mid Y\rangle_{g}\right) \in\left(A_{\Theta}^{\infty}\right)_{\mathrm{sa}}$.

Next, choose $r_{1}, \ldots, r_{n} \in \mathbb{R}$ so that $N\left(r_{1}, \ldots, r_{n}\right) \leq 1$. As $\nabla^{g}$ is compatible ${ }^{4}$ with $g$, we have

$$
\begin{aligned}
\left\|\sum_{j=1}^{n} r_{j} \cdot \partial_{j}\left(\langle X \mid Y\rangle_{g}\right)\right\|_{A_{\Theta}} & =\left\|\left\langle\nabla_{\sum_{j=1}^{n} r_{j} \cdot \partial_{j}}^{g}(X) \mid Y\right\rangle_{g}+\left\langle X \mid \nabla_{\sum_{j=1}^{n} r_{j} \cdot \partial_{j}}^{g}(Y)\right\rangle_{g}\right\|_{A_{\Theta}} \\
& \leq\left\|\left\langle\nabla_{\sum_{j=1}^{n} r_{j} \cdot \partial_{j}}^{g}(X) \mid Y\right\rangle_{g}\right\|_{A_{\Theta}}+\left\|\left\langle X \mid \nabla_{\sum_{j=1}^{n} r_{j} \cdot \partial_{j}}^{g}(Y)\right\rangle_{g}\right\|_{A_{\Theta}} \\
& \leq\left\|\nabla_{\sum_{j=1}^{n} r_{j} \cdot \partial_{j}}^{g}(X)\right\|_{g}\|Y\|_{g}+\|X\|_{g}\left\|\nabla_{\sum_{j=1}^{n} r_{j} \cdot \partial_{j}}^{g}(Y)\right\|_{g} \\
& \leq\|X\|\left\|_{g}\right\| \sum_{j=1}^{n} r_{j} \cdot \partial_{j}\|\|_{\mathcal{D}_{\Theta}}\|Y\|_{g}+\|X\|_{g}\|Y\|\left\|_{g}\right\| \sum_{j=1}^{n} r_{j} \cdot \partial_{j} \|_{\mathcal{D}_{\Theta}} \\
& \leq\|X\|\left\|_{g}\right\| Y\left\|_{g}+\right\| X\left\|_{g}\right\| Y \|_{g} \quad\left(\text { as } N\left(r_{1}, \ldots, r_{n}\right) \leq 1\right) \\
& \leq 2 \mathrm{D}_{g}(X) \mathrm{D}_{g}(Y) .
\end{aligned}
$$

\footnotetext{
${ }^{4}$ Metric compatibility is a requirement in the definition of a Levi-Civita connection.
} 
It is an easily-verified fact for any $C^{*}$-algebra $B$ that $\|\Re(b)\|_{B},\|\Im(b)\|_{B} \leq\|b\|_{B}$ for all $b \in B$, so

$$
\begin{aligned}
& \left\|\sum_{j=1}^{n} r_{j} \cdot \partial_{j}\left(\Re\left(\langle X \mid Y\rangle_{g}\right)\right)\right\|_{A_{\Theta}}=\left\|\Re\left(\sum_{j=1}^{n} r_{j} \cdot \partial_{j}\left(\langle X \mid Y\rangle_{g}\right)\right)\right\|_{A_{\Theta}} \leq 2 \mathrm{D}_{g}(X) \mathrm{D}_{g}(Y), \\
& \left\|\sum_{j=1}^{n} r_{j} \cdot \partial_{j}\left(\Im\left(\langle X \mid Y\rangle_{g}\right)\right)\right\|_{A_{\Theta}}=\left\|\Im\left(\sum_{j=1}^{n} r_{j} \cdot \partial_{j}\left(\langle X \mid Y\rangle_{g}\right)\right)\right\|_{A_{\Theta}} \leq 2 \mathrm{D}_{g}(X) \mathrm{D}_{g}(Y) .
\end{aligned}
$$

Therefore, as $r_{1}, \ldots, r_{n} \in \mathbb{R}$ are arbitrary subject to $N\left(r_{1}, \ldots, r_{n}\right) \leq 1$, an application of (4.1) yields

$$
\max \left(\mathrm{L}\left(\Re\left(\langle X \mid Y\rangle_{g}\right)\right), \mathrm{L}\left(\Im\left(\langle X \mid Y\rangle_{g}\right)\right)\right) \leq H\left(\mathrm{D}_{g}(X), \mathrm{D}_{g}(Y)\right)
$$

as required.

Proposition 4.11. $\mathrm{D}_{g}^{1}$ is $\|\cdot\|_{g}$-pre-compact in $\chi_{\Theta}$.

Proof. Fix $X=\sum_{j=1}^{n} a_{j} \bullet \mathbf{e}_{j} \in \mathrm{D}_{g}^{1}$, where $a_{1}, \ldots, a_{n} \in A_{\Theta}^{\infty}$.

Choose $r_{1}, \ldots, r_{n} \in \mathbb{R}$ satisfying $N\left(r_{1}, \ldots, r_{n}\right) \leq 1$, and let $\delta=\sum_{j=1}^{n} r_{j} \cdot \partial_{j}$. Then by the Leibniz rule,

$$
\begin{aligned}
\nabla_{\delta}^{g}(X) & =\sum_{j=1}^{n} \nabla_{\delta}^{g}\left(a_{j} \bullet \mathbf{e}_{j}\right)=\sum_{j=1}^{n}\left[\delta\left(a_{j}\right) \bullet \mathbf{e}_{j}+a_{j} \bullet \nabla_{\delta}^{g}\left(\mathbf{e}_{j}\right)\right] \\
& =\sum_{j=1}^{n} \delta\left(a_{j}\right) \bullet \mathbf{e}_{j}+\sum_{j=1}^{n} a_{j} \bullet \nabla_{\delta}^{g}\left(\mathbf{e}_{j}\right) .
\end{aligned}
$$

Rearranging the terms from both ends of (4.2) and then taking the norm of both sides, we obtain

$$
\begin{aligned}
\left\|\sum_{j=1}^{n} \delta\left(a_{j}\right) \bullet \mathbf{e}_{j}\right\|_{g} & =\left\|\nabla_{\delta}^{g}(X)-\sum_{j=1}^{n} a_{j} \bullet \nabla_{\delta}^{g}\left(\mathbf{e}_{j}\right)\right\| \leq\left\|\nabla_{\delta}^{g}(X)\right\|_{g}+\sum_{j=1}^{n}\left\|a_{j}\right\|_{A_{\Theta}}\left\|\nabla_{\delta}^{g}\left(\mathbf{e}_{j}\right)\right\|_{g} \\
& \leq\|X\|_{g}\|\delta\|_{\mathcal{D}_{\Theta}}+\sum_{j=1}^{n}\left\|a_{j}\right\|_{A_{\Theta}}\left\|\mathbf{e}_{j}\right\|\left\|_{g}\right\| \delta \|_{\mathcal{D}_{\Theta}} \\
& \leq 1+\sum_{j=1}^{n}\left\|a_{j}\right\|_{A_{\Theta}}\left\|\mathbf{e}_{j}\right\|_{g} \quad\left(\text { as }\|X\|_{g} \leq \mathrm{D}_{g}(X) \leq 1 \text { and }\|\delta\|_{\mathcal{D}_{\Theta}} \leq 1\right) .
\end{aligned}
$$

As $\|X\|_{g} \leq \mathrm{D}_{g}(X) \leq 1$, an application of Lemma 3.3 yields

$$
\left\|\sum_{j=1}^{n} a_{j} \bullet \mathbf{e}_{j}\right\|_{\text {st }}=\|X\|_{\text {st }} \leq\left\|\sqrt{g^{-1}}\right\|_{M_{n}\left(A_{\Theta}\right)}\|X\|_{g} \leq\left\|\sqrt{g^{-1}}\right\|_{M_{n}\left(A_{\Theta}\right)} .
$$

Hence, $\left\|a_{j}\right\|_{A_{\Theta}} \leq\left\|\sqrt{g^{-1}}\right\|_{M_{n}\left(A_{\Theta}\right)}$ for all $j \in[n]$, so letting $M \stackrel{\text { df }}{=} \max \left(\left\{\left\|\mathbf{e}_{j}\right\|_{g} \mid j \in[n]\right\}\right)$ gives us

$$
\left\|\sum_{j=1}^{n} \delta\left(a_{j}\right) \bullet \mathbf{e}_{j}\right\|_{g} \leq 1+n M\left\|\sqrt{g^{-1}}\right\|_{M_{n}\left(A_{\ominus}\right)} .
$$


It then follows from another application of Lemma 3.3 that

$$
\begin{aligned}
\left\|\sum_{j=1}^{n} \delta\left(a_{j}\right) \bullet \mathbf{e}_{j}\right\|_{\mathrm{st}} & \leq\left\|\sqrt{g^{-1}}\right\|_{M_{n}\left(A_{\Theta}\right)}\left\|\sum_{j=1}^{n} \delta\left(a_{j}\right) \bullet \mathbf{e}_{j}\right\|_{g} \\
& \leq\left\|\sqrt{g^{-1}}\right\|_{M_{n}\left(A_{\Theta}\right)}\left(1+n M\left\|\sqrt{g^{-1}}\right\|_{M_{n}\left(A_{\Theta}\right)}\right) .
\end{aligned}
$$

As $\Re\left(\delta\left(a_{j}\right)\right)=\delta\left(\Re\left(a_{j}\right)\right)$ and $\Im\left(\delta\left(a_{j}\right)\right)=\delta\left(\Im\left(a_{j}\right)\right)$ for all $j \in[n]$, we have

$$
\begin{aligned}
\forall j \in[n]: \quad\left\|\delta\left(\Re\left(a_{j}\right)\right)\right\|_{A_{\Theta}},\left\|\delta\left(\Im\left(a_{j}\right)\right)\right\|_{A_{\Theta}} & \leq\left\|\delta\left(a_{j}\right)\right\|_{A_{\Theta}} \\
& \leq\left\|\sqrt{g^{-1}}\right\|_{M_{n}\left(A_{\Theta}\right)}\left(1+n M\left\|\sqrt{g^{-1}}\right\|_{M_{n}\left(A_{\Theta}\right)}\right) .
\end{aligned}
$$

Recalling that $r_{1}, \ldots, r_{n} \in \mathbb{R}$ are arbitrary subject to $N\left(r_{1}, \ldots, r_{n}\right) \leq 1$, an application of (4.1) yields

$$
\forall j \in[n]: \quad \mathrm{L}\left(\Re\left(a_{j}\right)\right), \mathrm{L}\left(\Im\left(a_{j}\right)\right) \leq\left\|\sqrt{g^{-1}}\right\|_{M_{n}\left(A_{\Theta}\right)}\left(1+n M\left\|\sqrt{g^{-1}}\right\|_{M_{n}\left(A_{\Theta}\right)}\right) .
$$

Consequently,

$$
\begin{aligned}
& \forall j \in[n]: \\
& \Re\left(a_{j}\right), \Im\left(a_{j}\right) \in\left\{a \in \operatorname{Dom}(\mathrm{L}) \mid \mathrm{L}(a),\|a\|_{A_{\Theta}} \leq\left\|\sqrt{g^{-1}}\right\|_{M_{n}\left(A_{\Theta}\right)}\left(1+n M\left\|\sqrt{g^{-1}}\right\|_{M_{n}\left(A_{\Theta}\right)}\right)\right\},
\end{aligned}
$$

where the object on the right-hand side is a $\|\cdot\|_{A_{\Theta}}$-compact subset of $A_{\Theta}$ (see [5, Remark 2.46]). Hence, as $X \in \mathrm{D}_{g}^{1}$ is arbitrary, we have shown that there exists a single $\|\cdot\|_{A_{\Theta}}$-compact subset of $A_{\Theta}$ that contains the $A_{\Theta}$-coefficients of all elements of $\mathrm{D}_{g}^{1}$, which implies that $\mathrm{D}_{g}^{1}$ is $\|\cdot\|_{\mathrm{st}}$-precompact in $\chi_{\Theta}$. However, $\|\cdot\|_{\text {st }}$ and $\|\cdot\|_{g}$ are equivalent by Lemma 3.3, so $\mathrm{D}_{g}^{1}$ is $\|\cdot\|_{g}$-pre-compact in $\chi_{\Theta}$.

Definition $4.12([9])$. Let $A$ be a normed $\mathbb{C}$-vector space and $C$ a circled convex subset of $A$. The Minkowski gauge functional associated to $C$ is then the function $p$ that satisfies

$$
\begin{aligned}
& \operatorname{Dom}(p)=\left\{a \in A \mid \text { there exists an } r \in \mathbb{R}_{>0} \text { such that } a \in r \cdot C\right\} \\
& \forall a \in \operatorname{Dom}(p): \quad p(a) \stackrel{\text { df }}{=} \inf \left(\left\{r \in \mathbb{R}_{>0} \mid a \in r \cdot C\right\}\right) .
\end{aligned}
$$

Now, let $\mathrm{D}_{g, \mathrm{M}}$ denote the Minkowski gauge functional associated to $\overline{\mathrm{D}}_{g}^{1}\|\cdot\|_{g}$. Our objective is to show that $\left(\chi_{\Theta},\langle\cdot \mid \cdot\rangle_{g}, \mathrm{D}_{g, \mathrm{M}}, A_{\Theta}, \mathrm{L}\right)$ is an $(F, G, H)$-metrized quantum vector bundle, where $F$ is Leibniz, and $G$ and $H$ are defined as in Propositions 4.9 and 4.10 respectively. Before proceeding further, let us first collect some facts about Minkowski gauge functionals.

Lemma 4.13. Let $A$ be a normed $\mathbb{C}$-vector space, $B$ a $\mathbb{C}$-linear subspace of $A$, and $L$ a seminorm on $B$. Denote by $L_{\mathrm{M}}$ the Minkowski gauge functional associated to ${\overline{L^{1}}}^{\|\cdot\|_{A}}$. Then the following statements hold:

1) $L_{\mathrm{M}}^{r}={\overline{L^{r}}}^{\|\cdot\|_{A}}$ for all $r \in \mathbb{R}_{>0}$,

2) $B \subseteq \operatorname{Dom}\left(L_{\mathrm{M}}\right)$ and $L_{\mathrm{M}}(b) \leq L(b)$ for all $b \in B$,

3) if $a \in \operatorname{Dom}\left(L_{\mathrm{M}}\right)$, then there is a sequence $\left(b_{k}\right)_{k \in \mathbb{N}}$ in $B$ such that

- $L\left(b_{k}\right) \rightarrow L_{\mathrm{M}}(a)$ and

- $b_{k} \rightarrow a$ with respect to $\|\cdot\|_{A}$, 
4) $L \subseteq L_{\mathrm{M}}$ if $L$ is lower-semicontinuous on $\left(B,\|\cdot\|_{B}\right)$, where $\|\cdot\|_{B}$ denotes the restriction of $\|\cdot\|_{A}$ to $B$.

Proof. See [11, Proposition 4.4] and [13, Section 3].

With Lemma 4.13, we can make the following observations:

- $\mathrm{D}_{g, \mathrm{M}}$ satisfies property (3) of Definition 4.5.

We must first establish that $\mathrm{D}_{g, \mathrm{M}}$ is a norm on its domain. Let $X \in \operatorname{Dom}\left(\mathrm{D}_{g, \mathrm{M}}\right)$ satisfy $\mathrm{D}_{g, \mathrm{M}}(X)=0$. Then (3) of Lemma 4.13 tells us that there is a sequence $\left(X_{k}\right)_{k \in \mathbb{N}}$ in $\chi_{\Theta}^{\infty}$ such that $\mathrm{D}_{g}\left(X_{k}\right) \rightarrow \mathrm{D}_{g, \mathrm{M}}(X)=0$ and $X_{k} \rightarrow X$ with respect to $\|\cdot\|_{g}$, but $\left\|X_{k}\right\|_{g} \leq \mathrm{D}_{g}\left(X_{k}\right)$ by construction for every $k \in \mathbb{N}$, so $\left\|X_{k}\right\|_{g} \rightarrow 0$, or equivalently, $X_{k} \rightarrow 0_{\chi_{\Theta}}$ with respect to $\|\cdot\|_{g}$. Therefore, $X=0_{\chi_{\Theta}}$, but as Minkowski gauge functionals are already absolutely homogeneous, subadditive and non-negative, $\mathrm{D}_{g, \mathrm{M}}$ is indeed a norm on its domain.

Next, we have by (2) of Lemma 4.13 that $\chi_{\Theta}^{\infty} \subseteq \operatorname{Dom}\left(\mathrm{D}_{g, \mathrm{M}}\right)$. As $\chi_{\Theta}^{\infty}$ is a $\|\cdot\|_{g}$-dense $\mathbb{C}$-linear subspace of $\chi_{\Theta}$, so is $\operatorname{Dom}\left(\mathrm{D}_{g, \mathrm{M}}\right)$.

- $\mathrm{D}_{g, \mathrm{M}}$ satisfies property (4) of Definition 4.5.

Let $X \in \operatorname{Dom}\left(\mathrm{D}_{g, \mathrm{M}}\right)$. Then (3) of Lemma 4.13 tells us that there is a sequence $\left(X_{k}\right)_{k \in \mathbb{N}}$ in $\chi_{\Theta}^{\infty}$ such that $\mathrm{D}_{g}\left(X_{k}\right) \rightarrow \mathrm{D}_{g, \mathrm{M}}(X)$ and $X_{k} \rightarrow X$ with respect to $\|\cdot\|_{g}$, but, as before, $\left\|X_{k}\right\|_{g} \leq \mathrm{D}_{g}\left(X_{k}\right)$ by construction for every $k \in \mathbb{N}$, so $\|X\|_{g} \leq \mathrm{D}_{g, \mathrm{M}}(X)$.

- $\mathrm{D}_{g, \mathrm{M}}$ satisfies property (5) of Definition 4.5.

By (1) of Lemma 4.13, we have $\mathrm{D}_{g, \mathrm{M}}^{1}=\overline{\mathrm{D}}_{g}^{\|}\|\cdot\|_{g}$, so $\mathrm{D}_{g, \mathrm{M}}^{1}$ is $\|\cdot\|_{g}$-compact in $\chi_{\Theta}$ by Proposition 4.11 .

It remains to verify properties (6) and (7) of Definition 4.5, which we now turn our attention to.

Proposition 4.14. Let $a \in \operatorname{Dom}(\mathrm{L})$ and $X \in \operatorname{Dom}\left(\mathrm{D}_{g, \mathrm{M}}\right)$. Then $a \bullet X \in \operatorname{Dom}\left(\mathrm{D}_{g, \mathrm{M}}\right)$ and

$$
\mathrm{D}_{g, \mathrm{M}}(a \bullet X) \leq G\left(\|a\|_{A_{\Theta}}, \mathrm{L}(a), \mathrm{D}_{g, \mathrm{M}}(X)\right),
$$

where $G:[0, \infty)^{3} \rightarrow[0, \infty)$ is defined as in Proposition 4.9 .

Proof. By (3) of Lemma 4.13, there is a sequence $\left(X_{k}\right)_{k \in \mathbb{N}}$ in $\chi_{\Theta}^{\infty}$ such that

- $\mathrm{D}_{g}\left(X_{k}\right) \rightarrow \mathrm{D}_{g, \mathrm{M}}(X)$ and

- $X_{k} \rightarrow X$ with respect to $\|\cdot\|_{g}$.

Let $\mu$ denote the normalized Haar measure on $\mathbb{T}^{n}$. It is a well-known result of the theory of smooth vectors for a strongly continuous Lie-group action on a $C^{*}$-algebra that a net $\left(f_{\nu}\right)_{\nu \in \mathcal{N}}$ in $C_{c}^{\infty}\left(\mathbb{T}^{n}\right)$ exists such that

(a) $f_{\nu}$ is non-negative and $\int_{\mathbb{T}^{n}} f_{\nu}(\boldsymbol{s}) \mathrm{d} \mu(\boldsymbol{s})=1$ for all $\nu \in \mathcal{N}$,

(b) $a_{\nu} \stackrel{\mathrm{df}}{=} \int_{\mathbb{T}^{n}} f_{\nu}(\boldsymbol{s}) \cdot \alpha_{\boldsymbol{s}}(a) \mathrm{d} \mu(\boldsymbol{s}) \in A_{\Theta}^{\infty}$ for all $\nu \in \mathcal{N}$, and

(c) $a_{\nu} \rightarrow a$ with respect to $\|\cdot\|_{A_{\Theta}}$. 
In fact, as $f_{\nu}$ is non-negative for all $\nu \in \mathcal{N}$ and as self-adjoint elements of a $C^{*}$-algebra are preserved under $*$-homomorphisms, (b) can be strengthened to say that $a_{\nu} \in\left(A_{\Theta}^{\infty}\right)_{\text {sa }}$ for all $\nu \in \mathcal{N}$. Proceeding, we have for all $\nu \in \mathcal{N}$ and $\boldsymbol{t} \in \mathbb{T}^{n} \backslash\{\mathbf{0}\}$ that

$$
\begin{aligned}
& \frac{\left\|\alpha_{\boldsymbol{t}}\left(a_{\nu}\right)-a_{\nu}\right\|_{A_{\Theta}}}{\ell(\boldsymbol{t})}=\frac{\left\|\alpha_{\boldsymbol{t}}\left(\int_{\mathbb{T}^{n}} f_{\nu}(\boldsymbol{s}) \cdot \alpha_{\boldsymbol{s}}(a) \mathrm{d} \mu(\boldsymbol{s})\right)-\int_{\mathbb{T}^{n}} f_{\nu}(\boldsymbol{s}) \cdot \alpha_{\boldsymbol{s}}(a) \mathrm{d} \mu(\boldsymbol{s})\right\|_{A_{\Theta}}}{\ell(\boldsymbol{t})} \\
& =\frac{\left\|\int_{\mathbb{T}^{n}} f_{\nu}(\boldsymbol{s}) \cdot \alpha_{\boldsymbol{t}+\boldsymbol{s}}(a) \mathrm{d} \mu(\boldsymbol{s})-\int_{\mathbb{T}^{n}} f_{\nu}(\boldsymbol{s}) \cdot \alpha_{\boldsymbol{s}}(a) \mathrm{d} \mu(\boldsymbol{s})\right\|_{A_{\Theta}}}{\ell(\boldsymbol{t})} \\
& =\frac{\left\|\int_{\mathbb{T}^{n}} f_{\nu}(\boldsymbol{s}) \cdot \alpha_{\boldsymbol{s}+\boldsymbol{t}}(a) \mathrm{d} \mu(\boldsymbol{s})-\int_{\mathbb{T}^{n}} f_{\nu}(\boldsymbol{s}) \cdot \alpha_{\boldsymbol{s}}(a) \mathrm{d} \mu(\boldsymbol{s})\right\|_{A_{\Theta}}}{\ell(\boldsymbol{t})} \\
& =\frac{\left\|\int_{\mathbb{T}^{n}} f_{\nu}(\boldsymbol{s}) \cdot \alpha_{\boldsymbol{s}}\left(\alpha_{\boldsymbol{t}}(a)-a\right) \mathrm{d} \mu(\boldsymbol{s})\right\|_{A_{\Theta}}}{\ell(\boldsymbol{t})} \\
& \leq \frac{\int_{\mathbb{T}^{n}}\left\|f_{\nu}(\boldsymbol{s}) \cdot \alpha_{\boldsymbol{s}}\left(\alpha_{\boldsymbol{t}}(a)-a\right)\right\|_{A_{\Theta}} \mathrm{d} \mu(\boldsymbol{s})}{\ell(\boldsymbol{t})} \\
& =\frac{\int_{\mathbb{T}^{n}} f_{\nu}(s)\left\|\alpha_{\boldsymbol{s}}\left(\alpha_{\boldsymbol{t}}(a)-a\right)\right\|_{A_{\Theta}} \mathrm{d} \mu(\boldsymbol{s})}{\ell(\boldsymbol{t})} \quad\left(\text { as } f_{\nu} \text { is non-negative }\right) \\
& =\frac{\int_{\mathbb{T}^{n}} f_{\nu}(s)\left\|\alpha_{\boldsymbol{t}}(a)-a\right\|_{A_{\Theta}} \mathrm{d} \mu(\boldsymbol{s})}{\ell(\boldsymbol{t})} \quad\left(\text { as } \alpha_{\boldsymbol{s}} \text { is isometric }\right) \\
& =\frac{\left\|\alpha_{\boldsymbol{t}}(a)-a\right\|_{A_{\Theta}}}{\ell(\boldsymbol{t})} \quad\left(\operatorname{as} \int_{\mathbb{T}^{n}} f_{\nu}(\boldsymbol{s}) \mathrm{d} \mu(\boldsymbol{s})=1\right) .
\end{aligned}
$$

Consequently, $a_{\nu} \in \operatorname{Dom}(\mathrm{L})$ and $\mathrm{L}\left(a_{\nu}\right) \leq \mathrm{L}(a)$ for all $\nu \in \mathcal{N}$. Invoking Proposition 4.9, we get

$$
\begin{aligned}
\forall \nu \in \mathcal{N}, \forall k \in \mathbb{N}: \quad \mathrm{D}_{g}\left(a_{\nu} \bullet X_{k}\right) & \leq G\left(\left\|a_{\nu}\right\|_{A_{\Theta}}, \mathrm{L}\left(a_{\nu}\right), \mathrm{D}_{g}\left(X_{k}\right)\right) \\
& \leq G\left(\left\|a_{\nu}\right\|_{A_{\Theta}}, \mathrm{L}(a), \mathrm{D}_{g}\left(X_{k}\right)\right),
\end{aligned}
$$

where the last inequality is due to the fact that $G$ is non-decreasing in each argument. By (2) of Lemma 4.13, $Y \in \operatorname{Dom}\left(\mathrm{D}_{g, \mathrm{M}}\right)$ and $\mathrm{D}_{g, \mathrm{M}}(Y) \leq \mathrm{D}_{g}(Y)$ for all $Y \in \chi_{\Theta}^{\infty}$, so

$$
\forall \nu \in \mathcal{N}, \forall k \in \mathbb{N}: \quad \mathrm{D}_{g, \mathrm{M}}\left(a_{\nu} \bullet X_{k}\right) \leq G\left(\left\|a_{\nu}\right\|_{A_{\Theta}}, \mathrm{L}(a), \mathrm{D}_{g}\left(X_{k}\right)\right) .
$$

Let $\epsilon>0$. As the right-hand side of this inequality converges to $G\left(\|a\|_{A_{\Theta}}, \mathrm{L}(a), \mathrm{D}_{g, \mathrm{M}}(X)\right)$ by the continuity of $G$, we find for all $\nu \in \mathcal{N}$ and $k \in \mathbb{N}$ sufficiently large that

$$
\mathrm{D}_{g, \mathrm{M}}\left(a_{\nu} \bullet X_{k}\right) \leq G\left(\|a\|_{A_{\Theta}}, \mathrm{L}(a), \mathrm{D}_{g, \mathrm{M}}(X)\right)+\epsilon .
$$

As $a_{\nu} \bullet X_{k} \rightarrow a \bullet X$ with respect to $\|\cdot\|_{g}$, it follows from the closure clause implicit in (1) of Lemma 4.13 that $a \bullet X \in \operatorname{Dom}\left(\mathrm{D}_{g, \mathrm{M}}\right)$ and

$$
\mathrm{D}_{g, \mathrm{M}}(a \bullet X) \leq G\left(\|a\|_{A_{\Theta}}, \mathrm{L}(a), \mathrm{D}_{g, \mathrm{M}}(X)\right)+\epsilon .
$$

However, $\epsilon>0$ is arbitrary, so

$$
\mathrm{D}_{g, \mathrm{M}}(a \bullet X) \leq G\left(\|a\|_{A_{\Theta}}, \mathrm{L}(a), \mathrm{D}_{g, \mathrm{M}}(X)\right)
$$

as required. 
Proposition 4.15. Let $X, Y \in \operatorname{Dom}\left(\mathrm{D}_{g, \mathrm{M}}\right)$. Then $\Re\left(\langle X \mid Y\rangle_{g}\right), \Im\left(\langle X \mid Y\rangle_{g}\right) \in \operatorname{Dom}(\mathrm{L})$ and $\max \left(\mathrm{L}\left(\Re\left(\langle X \mid Y\rangle_{g}\right)\right), \mathrm{L}\left(\Im\left(\langle X \mid Y\rangle_{g}\right)\right)\right) \leq H\left(\mathrm{D}_{g, \mathrm{M}}(X), \mathrm{D}_{g, \mathrm{M}}(Y)\right)$,

where $H:[0, \infty)^{2} \rightarrow[0, \infty)$ is defined as in Proposition 4.10 .

Proof. By (3) of Lemma 4.13, there are sequences $\left(X_{k}\right)_{k \in \mathbb{N}}$ and $\left(Y_{k}\right)_{k \in \mathbb{N}}$ in $\chi_{\Theta}^{\infty}$ such that

- $\mathrm{D}_{g}\left(X_{k}\right) \rightarrow \mathrm{D}_{g, \mathrm{M}}(X)$ and $\mathrm{D}_{g}\left(Y_{k}\right) \rightarrow \mathrm{D}_{g, \mathrm{M}}(Y)$, and

- $X_{k} \rightarrow X$ and $Y_{k} \rightarrow Y$ with respect to $\|\cdot\|_{g}$.

We already have from Proposition 4.10 that

$$
\forall k \in \mathbb{N}: \quad \max \left(\mathrm{L}\left(\Re\left(\left\langle X_{k} \mid Y_{k}\right\rangle_{g}\right)\right), \mathrm{L}\left(\Im\left(\left\langle X_{k} \mid Y_{k}\right\rangle_{g}\right)\right)\right) \leq H\left(\mathrm{D}_{g}\left(X_{k}\right), \mathrm{D}_{g}\left(Y_{k}\right)\right) .
$$

Let $\epsilon>0$. As the right-hand side of this inequality converges to $H\left(\mathrm{D}_{g, \mathrm{M}}(X), \mathrm{D}_{g, \mathrm{M}}(Y)\right)$ by the continuity of $H$, we find for all $k \in \mathbb{N}$ sufficiently large that

$$
\max \left(\mathrm{L}\left(\Re\left(\left\langle X_{k} \mid Y_{k}\right\rangle_{g}\right)\right), \mathrm{L}\left(\Im\left(\left\langle X_{k} \mid Y_{k}\right\rangle_{g}\right)\right)\right) \leq H\left(\mathrm{D}_{g, \mathrm{M}}(X), \mathrm{D}_{g, \mathrm{M}}(Y)\right)+\epsilon .
$$

As $\Re\left(\left\langle X_{k} \mid Y_{k}\right\rangle_{g}\right) \rightarrow \Re\left(\langle X \mid Y\rangle_{g}\right)$ and $\Im\left(\left\langle X_{k} \mid Y_{k}\right\rangle_{g}\right) \rightarrow \Im\left(\langle X \mid Y\rangle_{g}\right)$ with respect to $\|\cdot\|_{A_{\Theta}}$, the closure clause implicit in property (5) of Definition 4.2 says that $\Re\left(\langle X \mid Y\rangle_{g}\right), \Im\left(\langle X \mid Y\rangle_{g}\right) \in$ $\operatorname{Dom}(\mathrm{L})$ and

$$
\max \left(\mathrm{L}\left(\Re\left(\langle X \mid Y\rangle_{g}\right)\right), \mathrm{L}\left(\Im\left(\langle X \mid Y\rangle_{g}\right)\right)\right) \leq H\left(\mathrm{D}_{g, \mathrm{M}}(X), \mathrm{D}_{g, \mathrm{M}}(Y)\right)+\epsilon .
$$

However, $\epsilon>0$ is arbitrary, so

$$
\max \left(\mathrm{L}\left(\Re\left(\langle X \mid Y\rangle_{g}\right)\right), \mathrm{L}\left(\Im\left(\langle X \mid Y\rangle_{g}\right)\right)\right) \leq H\left(\mathrm{D}_{g, \mathrm{M}}(X), \mathrm{D}_{g, \mathrm{M}}(Y)\right)
$$

as required.

Theorem 4.16. $\left(\chi_{\Theta},\langle\cdot \mid \cdot\rangle_{g}, \mathrm{D}_{g, \mathrm{M}}, A_{\Theta}, \mathrm{L}\right)$ is an $(F, G, H)$-metrized quantum vector bundle, where $F$ is Leibniz, and $G$ and $H$ are defined as in Propositions 4.9 and 4.10 respectively.

Remark 4.17. We could have worked with more general $C^{*}$-algebras in this paper, but here are reasons why we focus only on generically transcendental quantum tori:

1) quantum tori are a convenient source of compact quantum metric spaces,

2) the norm $\|\cdot\|_{\mathcal{D}_{\Theta}}$ on the space of $*$-derivations on $A_{\Theta}^{\infty}$ enables us to conveniently prove the inequalities necessary to achieve the objective of this paper,

3) by Theorem 3.6, we have a single parameter for the D-norms on our metrized quantum vector bundles.

\section{A distance-zero result for the modular Gromov-Hausdorff propinquity}

In [6], Latrémolière introduced the modular Gromov-Hausdorff propinquity as a means of measuring, for an admissible triple $(F, G, H)$, how close two $(F, G, H)$-metrized quantum vector bundles are to each other, in the sense of how close they are to being isomorphic, in terms of their Hilbert- $C^{*}$-module structures and their metric structures.

Our aim in this section is to prove the following zero-distance result. 
Theorem 5.1. Let $g$ be a Riemannian metric, and let $r, s \in \mathbb{R}_{>0}$. Then $\Lambda_{F, G, H}^{\bmod }\left(\Omega_{r}, \Omega_{s}\right)=0$, where

$$
\Omega_{r} \stackrel{\mathrm{df}}{=}\left(\chi_{\Theta},\langle\cdot \mid \cdot\rangle_{r \cdot g}, \mathrm{D}_{r \cdot g, \mathrm{M}}, A_{\Theta}, \mathrm{L}\right) \quad \text { and } \quad \Omega_{s} \stackrel{\mathrm{df}}{=}\left(\chi_{\Theta},\langle\cdot \mid \cdot\rangle_{s \cdot g}, \mathrm{D}_{s \cdot g, \mathrm{M}}, A_{\Theta}, \mathrm{L}\right),
$$

$F$ is Leibniz, and $G$ and $H$ are defined as in Propositions 4.9 and 4.10 respectively.

Definition $5.2([6])$. Let $B$ be a unital $C^{*}$-algebra, and let $\mathfrak{b} \in B_{\mathrm{sa}}$. The 1 -level set of $\mathfrak{b}$ is then the subset $\mathscr{S}(B \mid \mathfrak{b})$ of $\mathscr{S}(B)$ defined by

$$
\mathscr{S}(B \mid \mathfrak{b}) \stackrel{\mathrm{df}}{=}\left\{\phi \in \mathscr{S}(B) \mid \phi\left(\left(1_{B}-\mathfrak{b}\right)^{*}\left(1_{B}-\mathfrak{b}\right)\right)=0=\phi\left(\left(1_{B}-\mathfrak{b}\right)\left(1_{B}-\mathfrak{b}\right)^{*}\right)\right\} .
$$

Definition $5.3([6])$. Let $(F, G, H)$ be an admissible triple. An $(F, G, H)$-modular bridge is then an ordered 9-tuple $\gamma=\left(\Omega_{1}, \Omega_{2}, B, \mathfrak{b}, \pi_{1}, \pi_{2}, \mathcal{N}, \alpha, \beta\right)$ satisfying the following six properties:

1) $\Omega_{1}$ and $\Omega_{2}$ are $(F, G, H)$-metrized quantum vector bundles, ${ }^{5}$

2) $B$ is a unital $C^{*}$-algebra,

$3) \mathfrak{b}$ is an element of $B_{\text {sa }}$, called the pivot, such that $\mathscr{S}(B \mid \mathfrak{b}) \neq \varnothing$ and $\|\mathfrak{b}\|_{B}=1$,

4) $\pi_{1}$ and $\pi_{2}$ are, respectively, unital *-monomorphisms from $A_{1}$ and $A_{2}$ to $B$,

5) $\mathcal{N}$ is a non-empty set,

6) $\alpha$ and $\beta$ are, respectively, functions from $\mathcal{N}$ to $D_{1}^{1}$ and $D_{2}^{1}$.

The domain of $\gamma$, denoted by $\operatorname{Dom}(\gamma)$, is defined to be $\Omega_{1}$, and the co-domain of $\gamma$, denoted by $\operatorname{Co-} \operatorname{Dom}(\gamma)$, is defined to be $\Omega_{2}$. We say that $\left(\Omega_{1}, \Omega_{2}, B, \mathfrak{b}, \pi_{1}, \pi_{2}, \mathcal{N}, \alpha, \beta\right)$ is a modular bridge from $\Omega_{1}$ to $\Omega_{2}$.

A modular bridge between two metrized quantum vector bundles yields useful numerical quantities that indicate how close the metrized quantum vector bundles are to each other. Before defining these quantities, we require a preliminary definition.

Definition $5.4([6])$. Let $\Omega=(\mathrm{X},\langle\cdot \mid \cdot\rangle, \mathrm{D}, A, \mathrm{~L})$ be a metrized quantum vector bundle. The modular Monge-Kantorovich metric of $\Omega$ is then the metric ${ }^{6} \mathrm{k}_{\Omega}$ on $\mathrm{X}$ defined by

$$
\forall \zeta, \eta \in \mathrm{X}: \quad \mathrm{k}_{\Omega}(\zeta, \eta) \stackrel{\mathrm{df}}{=} \sup \left(\left\{\|\langle\zeta-\eta \mid \theta\rangle\|_{\langle\cdot \mid \cdot\rangle} \mid \theta \in \mathrm{D}^{1}\right\}\right) .
$$

Definition 5.5 ([6]). Let $(F, G, H)$ be an admissible triple and

$$
\gamma=\left(\Omega_{1}, \Omega_{2}, B, \mathfrak{b}, \pi_{1}, \pi_{2}, \mathcal{N}, \alpha, \beta\right)
$$

an $(F, G, H)$-modular bridge.

1. The bridge seminorm of $\gamma$ is the seminorm $\mathrm{bn}_{\gamma}$ on $A_{1} \oplus A_{2}$ defined by

$$
\forall a_{1} \in A_{1}, \forall a_{2} \in A_{2}: \quad \operatorname{bn}_{\gamma}\left(a_{1}, a_{2}\right) \stackrel{\mathrm{df}}{=}\left\|\pi_{1}\left(a_{1}\right) \mathfrak{b}-\mathfrak{b} \pi_{2}\left(a_{2}\right)\right\|_{B} .
$$

2. The basic reach $\rho_{b}(\gamma)$ of $\gamma$ is the Hausdorff distance, in the seminormed space $\left(A_{1} \oplus A_{2}, \mathrm{bn}_{\gamma}\right)$, between the embedded images of $\operatorname{Dom}\left(L_{1}\right)$ and $\operatorname{Dom}\left(L_{2}\right)$, i.e.,

$$
\rho_{b}(\gamma) \stackrel{\mathrm{df}}{=} \max \left(\sup _{\substack{a \\ a_{1} \in \operatorname{Dom}\left(\mathrm{L}_{1}\right) \\ \mathrm{L}_{1}\left(a_{1}\right) \leq 1}} \inf _{\substack{a_{2} \in \operatorname{Dom}\left(\mathrm{L}_{2}\right) \\ \mathrm{L}_{2}\left(a_{2}\right) \leq 1}} \mathrm{bn}_{\gamma}\left(a_{1}, a_{2}\right), \sup _{\substack{a_{2} \in \operatorname{Dom}\left(\mathrm{L}_{2}\right) \\ \mathrm{L}_{2}\left(a_{2}\right) \leq 1}} \inf _{\substack{a_{1} \in \operatorname{Dom}\left(\mathrm{L}_{1}\right) \\ \mathrm{L}_{1}\left(a_{1}\right) \leq 1}} \mathrm{bn}_{\gamma}\left(a_{1}, a_{2}\right)\right) .
$$

\footnotetext{
${ }^{5}$ Note. From now on, we will write $\Omega_{1}=\left(\mathrm{X}_{1},\langle\cdot \mid \cdot\rangle_{1}, \mathrm{D}_{1}, A_{1}, \mathrm{~L}_{1}\right)$ and $\Omega_{2}=\left(\mathrm{X}_{2},\langle\cdot \mid \cdot\rangle_{2}, \mathrm{D}_{2}, A_{2}, \mathrm{~L}_{2}\right)$.

${ }^{6}$ This is a legitimate metric, thanks to property (5) of Definition 4.5 .
} 
3. The height of $\gamma$ is the non-negative quantity $\varsigma(\gamma)$ defined by

$$
\varsigma(\gamma) \stackrel{\mathrm{df}}{=} \max \left(\begin{array}{l}
\operatorname{Haus}_{\mathrm{mk}_{\mathrm{L}_{1}}}\left(\mathscr{S}\left(A_{1}\right),\left\{\phi \circ \pi_{1} \mid \phi \in \mathscr{S}(B \mid \mathfrak{b})\right\}\right) \\
\operatorname{Haus}_{\mathrm{mk}_{\mathrm{L}_{2}}}\left(\mathscr{S}\left(A_{2}\right),\left\{\psi \circ \pi_{2} \mid \psi \in \mathscr{S}(B \mid \mathfrak{b})\right\}\right)
\end{array}\right),
$$

where

- Haus $\mathrm{mk}_{\mathrm{L}_{1}}$ denotes the $\mathrm{mk}_{\mathrm{L}_{1}}$-induced Hausdorff distance between subsets of $\mathscr{S}\left(A_{1}\right)$, and

- Haus $\mathrm{mk}_{\mathrm{L}_{2}}$ denotes the $\mathrm{mk}_{\mathrm{L}_{2}}$-induced Hausdorff distance between subsets of $\mathscr{S}\left(A_{2}\right)$.

4. The deck seminorm of $\gamma$ is the seminorm $\mathrm{dn}_{\gamma}$ on $\mathbf{X}_{1} \oplus \mathbf{X}_{2}$ defined by

$$
\begin{aligned}
& \forall \zeta \in \mathrm{X}_{1}, \forall \eta \in \mathrm{X}_{2}: \\
& \operatorname{dn}_{\gamma}(\zeta, \eta) \stackrel{\mathrm{df}}{=} \sup \left(\left\{\operatorname{bn}_{\gamma}\left(\langle\zeta \mid \alpha(\nu)\rangle_{1},\langle\eta \mid \beta(\nu)\rangle_{2}\right), \operatorname{bn}_{\gamma}\left(\langle\alpha(\nu) \mid \zeta\rangle_{1},\langle\beta(\nu) \mid \eta\rangle_{2}\right) \mid \nu \in \mathcal{N}\right\}\right) .
\end{aligned}
$$

5. The modular reach of $\gamma$ is the non-negative quantity $\rho^{\sharp}(\gamma)$ defined by

$$
\rho^{\sharp}(\gamma) \stackrel{\mathrm{df}}{=} \sup \left(\left\{\mathrm{dn}_{\gamma}(\alpha(\nu), \beta(\nu)) \mid \nu \in \mathcal{N}\right\}\right) .
$$

6. The imprint of $\gamma$ is the non-negative quantity $\varpi(\gamma)$ defined by

$$
\varpi(\gamma) \stackrel{\text { df }}{=} \max \left(\operatorname{Haus}_{\mathrm{k}_{1}}\left(\operatorname{Range}(\alpha), \mathrm{D}_{1}^{1}\right), \operatorname{Haus}_{\mathrm{k}_{\Omega_{2}}}\left(\operatorname{Range}(\beta), \mathrm{D}_{2}^{1}\right)\right),
$$

where

- Haus $\mathrm{k}_{\Omega_{1}}$ denotes the $\mathrm{k}_{\Omega_{1}}$-induced Hausdorff distance between subsets of $\mathrm{X}_{1}$, and

- Haus $_{\mathrm{k}_{2}}$ denotes the $\mathrm{k}_{\Omega_{2}}$-induced Hausdorff distance between subsets of $\mathrm{X}_{2}$.

7. The reach of $\gamma$ is the non-negative quantity $\rho(\gamma)$ defined by

$$
\rho(\gamma) \stackrel{\mathrm{df}}{=} \max \left(\rho_{b}(\gamma), \rho^{\sharp}(\gamma)+\varpi(\gamma)\right) .
$$

8. The length of $\gamma$ is the non-negative quantity $\lambda(\gamma)$ defined by

$$
\lambda(\gamma) \stackrel{\mathrm{df}}{=} \max (\varsigma(\gamma), \rho(\gamma))
$$

Remark 5.6. Lemma 4.19 of [6] establishes that the numerical quantities defined in Definition 5.5 are finite.

Definition 5.7 ([6]). The modular Gromov-Hausdorff propinquity, for an admissible triple $(F, G, H)$, is the (class) function $\Lambda_{F, G, H}^{\text {mod }}$ from the class of all ordered pairs of $(F, G, H)$-metrized quantum vector bundles to $[0, \infty)$ defined by

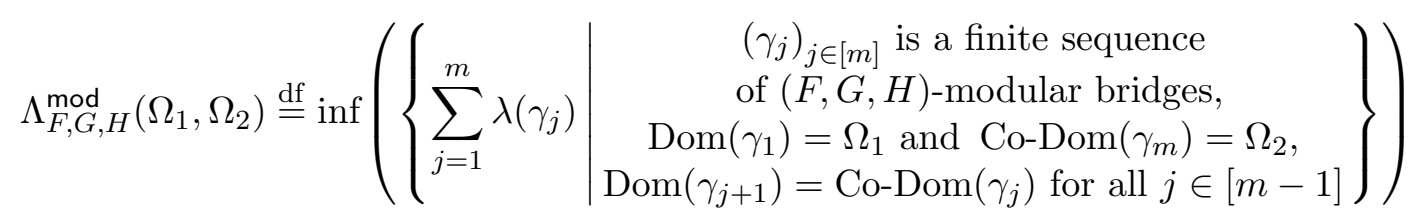

for all $(F, G, H)$-metrized quantum vector bundles $\Omega_{1}$ and $\Omega_{2}$.

We now define a full quantum isometry between metrized quantum vector bundles. 
Definition $5.8([6])$. Let $(F, G, H)$ be an admissible triple. Let $\Omega_{1}$ and $\Omega_{2}$ be $(F, G, H)$-metrized quantum vector bundles. A full quantum isometry from $\Omega_{1}$ to $\Omega_{2}$ is then an ordered pair $(\mathscr{L}, \mathscr{D})$ with the following properties:

- $\mathscr{L}$ is a unital $*$-isomorphism from $A_{1}$ to $A_{2}$,

- $\mathscr{D}$ is a continuous linear isomorphism (not necessarily unitary) from $\mathrm{X}_{1}$ to $\mathrm{X}_{2}$,

- $\mathrm{L}_{2} \circ \mathscr{L}=\mathrm{L}_{1}$,

- $\mathscr{D}(a \bullet \zeta)=\mathscr{L}(a) \bullet \mathscr{D}(\zeta)$ for all $a \in A_{1}$ and $\zeta \in \mathrm{X}_{1}$,

- $\mathrm{D}_{2} \circ \mathscr{D}=\mathrm{D}_{1}$,

- $\langle\mathscr{D}(\cdot) \mid \mathscr{D}(\cdot)\rangle_{2}=\mathscr{L} \circ\langle\cdot \mid \cdot\rangle_{1}$.

The following theorem says that the modular Gromov-Hausdorff propinquity has the required properties to qualify as a (class) pseudometric.

Theorem $5.9([6])$. Let $(F, G, H)$ be an admissible triple. For all $(F, G, H)$-metrized quantum vector bundles $\Omega_{1}, \Omega_{2}$ and $\Omega_{3}$, the following statements hold:

1) $\Lambda_{F, G, H}^{\text {mod }}\left(\Omega_{1}, \Omega_{2}\right)=\Lambda_{F, G, H}^{\text {mod }}\left(\Omega_{2}, \Omega_{1}\right)$,

2) $\Lambda_{F, G, H}^{\text {mod }}\left(\Omega_{1}, \Omega_{3}\right) \leq \Lambda_{F, G, H}^{\bmod }\left(\Omega_{1}, \Omega_{2}\right)+\Lambda_{F, G, H}^{\bmod }\left(\Omega_{2}, \Omega_{3}\right)$,

3) $\Lambda_{F, G, H}^{\mathrm{mod}}\left(\Omega_{1}, \Omega_{2}\right)=0$ if and only if there exists a full quantum isometry from $\Omega_{1}$ to $\Omega_{2}$.

Lemma 5.10. Let $g$ be a Riemannian metric, and let $r, s \in \mathbb{R}_{>0}$. Then

$$
\operatorname{Dom}\left(\mathrm{D}_{r \cdot g, \mathrm{M}}\right)=\operatorname{Dom}\left(\mathrm{D}_{s \cdot g, \mathrm{M}}\right) \quad \text { and } \quad \frac{1}{\sqrt{r}} \mathrm{D}_{r \cdot g, \mathrm{M}}=\frac{1}{\sqrt{s}} \mathrm{D}_{s \cdot g, \mathrm{M}} .
$$

Proof. One can verify that $\nabla^{r \cdot g}$ is also a Levi-Civita connection for $g$, so $\nabla^{r \cdot g}=\nabla^{g}$ by Theorem 3.6 and

$$
\forall \delta \in \mathcal{D}_{\Theta}, \forall X, Y \in \chi_{\Theta}^{\infty}: \quad\left\langle\nabla_{\delta}^{r \cdot g}(X) \mid Y\right\rangle_{r \cdot g}=\left\langle\nabla_{\delta}^{g}(X) \mid Y\right\rangle_{r \cdot g}=r \cdot\left\langle\nabla_{\delta}^{g}(X) \mid Y\right\rangle_{g},
$$

which yields

$$
\forall \delta \in \mathcal{D}_{\Theta}, \forall X, Y \in \chi_{\Theta}^{\infty}: \quad\left\|\left\langle\nabla_{\delta}^{r \cdot g}(X) \mid Y\right\rangle_{r \cdot g}\right\|_{A_{\Theta}}=\sqrt{r}\left\|\left\langle\nabla_{\delta}^{g}(X) \mid \sqrt{r} \cdot Y\right\rangle_{g}\right\|_{A_{\Theta}} .
$$

Hence, we have for all $\delta \in \mathcal{D}_{\Theta}$ and $X \in \chi_{\Theta}^{\infty}$ that

$$
\begin{aligned}
\left\|\nabla_{\delta}^{r \cdot g}(X)\right\|_{r \cdot g} & =\sup _{\substack{Y \in \chi_{\Theta} \\
\|Y\|_{r \cdot g}=1}}\left\|\left\langle\nabla_{\delta}^{r \cdot g}(X) \mid Y\right\rangle_{r \cdot g}\right\|_{A_{\Theta}}=\sup _{\substack{Y \in \chi_{\Theta} \\
\|Y\|_{r \cdot g}=1}} \sqrt{r}\left\|\left\langle\nabla_{\delta}^{g}(X) \mid \sqrt{r} \cdot Y\right\rangle_{g}\right\|_{A_{\Theta}} \\
= & \sup _{\substack{Y \in \chi_{\Theta} \\
\|\sqrt{r} \cdot Y\|_{g}=1}} \sqrt{r}\left\|\left\langle\nabla_{\delta}^{g}(X) \mid \sqrt{r} \cdot Y\right\rangle_{g}\right\|_{A_{\Theta}}\left(\text { as }\|\cdot\|_{r \cdot g}=\sqrt{r}\|\cdot\|_{g}\right) \\
= & \sup _{\substack{Y \in \chi_{\Theta} \\
\|Y\|_{g}=1}} \sqrt{r}\left\|\left\langle\nabla_{\delta}^{g}(X) \mid Y\right\rangle_{g}\right\|_{A_{\Theta}}=\sqrt{r}\left\|\nabla_{\delta}^{g}(X)\right\|_{g} .
\end{aligned}
$$

It follows readily that

$$
\forall X \in \chi_{\Theta}^{\infty}: \quad\|X\|_{r \cdot g}=\sup _{\substack{\delta \in \mathcal{D}_{\Theta} \\\|\delta\|_{\mathcal{D}_{\Theta}} \leq 1}}\left\|\nabla_{\delta}^{r \cdot g}(X)\right\|_{r \cdot g}=\sqrt{r} \sup _{\substack{\delta \in \mathcal{D}_{\Theta} \\\|\delta\|_{\mathcal{D}_{\Theta}} \leq 1}}\left\|\nabla_{\delta}^{g}(X)\right\|_{g}=\sqrt{r}\|X\|_{g} .
$$


We thus obtain $\|\cdot\|_{r \cdot g}=\sqrt{r}\|\cdot\|_{g}$, so

$$
\mathrm{D}_{r \cdot g}=\max \left(\|\cdot\|_{r \cdot g},\|\cdot\|_{r \cdot g}\right)=\max \left(\sqrt{r}\|\cdot\|_{g}, \sqrt{r}\|\cdot\|_{g}\right)=\sqrt{r} \max \left(\|\cdot\|_{g},\|\cdot\| \|_{g}\right)=\sqrt{r} \mathrm{D}_{g} .
$$

Consequently, $\mathrm{D}_{g}^{1}=\mathrm{D}_{r \cdot g}^{\sqrt{r}}=\sqrt{r} \cdot \mathrm{D}_{r \cdot g}^{1}$.

Now, we have by Definition 4.12 that

$$
\begin{aligned}
& \operatorname{Dom}\left(\mathrm{D}_{g, \mathrm{M}}\right)=\sup \left(\left\{X \in \chi_{\Theta} \mid \text { there exists a } t \in \mathbb{R}_{>0} \text { such that } X \in t \cdot \overline{\mathrm{D}}_{g}^{1}\|\cdot\|_{g}\right\}\right) \\
& =\sup \left(\left\{X \in \chi_{\Theta} \mid \text { there exists a } t \in \mathbb{R}_{>0} \text { such that } X \in t \cdot \overline{\sqrt{r} \cdot \mathrm{D}_{r \cdot g}^{1}}\|\cdot\|_{g}\right\}\right) \\
& =\sup \left(\left\{X \in \chi_{\Theta} \mid \text { there exists a } t \in \mathbb{R}_{>0} \text { such that } X \in t \sqrt{r} \cdot \overline{\mathrm{D}_{r \cdot g}^{1}}\|\cdot\|_{g}\right\}\right) \\
& =\sup \left(\left\{X \in \chi_{\Theta} \mid \text { there exists a } t \in \mathbb{R}_{>0} \text { such that } X \in t \sqrt{r} \cdot \overline{\mathrm{D}_{r \cdot g}^{1}}\|\cdot\|_{r \cdot g}\right\}\right) \\
& \text { (as }\|\cdot\|_{g} \text { and }\|\cdot\|_{r \cdot g} \text { are equivalent) } \\
& =\sup \left(\left\{X \in \chi_{\Theta} \mid \text { there exists a } t \in \mathbb{R}_{>0} \text { such that } X \in t \cdot \overline{\mathrm{D}_{r \cdot g}^{1}}\|\cdot\|_{r \cdot g}\right\}\right) \\
& =\operatorname{Dom}\left(\mathrm{D}_{r \cdot g, \mathrm{M}}\right) \text {. }
\end{aligned}
$$

Next, observe for all $X \in \operatorname{Dom}\left(\mathrm{D}_{g, \mathrm{M}}\right)=\operatorname{Dom}\left(\mathrm{D}_{r \cdot g, \mathrm{M}}\right)$ that

$$
\begin{aligned}
\mathrm{D}_{g, \mathrm{M}}(X) & =\inf \left(\left\{t \in \mathbb{R}_{>0} \mid X \in t \cdot \overline{\mathrm{D}_{g}^{1}}\|\cdot\|_{g}\right\}\right) \\
& =\inf \left(\left\{t \in \mathbb{R}_{>0} \mid X \in t \cdot \overline{\sqrt{r} \cdot \mathrm{D}_{r \cdot g}^{1}}\|\cdot\|_{g}\right\}\right) \\
& =\inf \left(\left\{t \in \mathbb{R}_{>0} \mid X \in t \sqrt{r} \cdot \overline{\mathrm{D}_{r \cdot g}^{1}}\|\cdot\|_{g}\right\}\right) \\
& =\inf \left(\left\{t \in \mathbb{R}_{>0} \mid X \in t \sqrt{r} \cdot \overline{\mathrm{D}_{r \cdot g}^{1}}\|\cdot\|_{r \cdot g}\right\}\right) \quad\left(\text { as }\|\cdot\|_{g} \text { and }\|\cdot\|_{r \cdot g} \text { are equivalent }\right) \\
& =\frac{1}{\sqrt{r}} \inf \left(\left\{t \in \mathbb{R}_{>0} \mid X \in t \cdot \overline{\mathrm{D}_{r \cdot g}^{1}}\|\cdot\|_{r \cdot g}\right\}\right) \\
& =\frac{1}{\sqrt{r}} \mathrm{D}_{r \cdot g, \mathrm{M}}(X) .
\end{aligned}
$$

As $X \in \operatorname{Dom}\left(\mathrm{D}_{g, \mathrm{M}}\right)=\operatorname{Dom}\left(\mathrm{D}_{r \cdot g, \mathrm{M}}\right)$ is arbitrary, we obtain $\mathrm{D}_{r \cdot g, \mathrm{M}}=\sqrt{r} \mathrm{D}_{g, \mathrm{M}}$.

Similarly, $\operatorname{Dom}\left(\mathrm{D}_{g, \mathrm{M}}\right)=\operatorname{Dom}\left(\mathrm{D}_{s \cdot g, \mathrm{M}}\right)$ and $\mathrm{D}_{s \cdot g, \mathrm{M}}=\sqrt{s} \mathrm{D}_{g, \mathrm{M}}$, so

$$
\operatorname{Dom}\left(\mathrm{D}_{r \cdot g, \mathrm{M}}\right)=\operatorname{Dom}\left(\mathrm{D}_{s \cdot g, \mathrm{M}}\right) \quad \text { and } \quad \frac{1}{\sqrt{r}} \mathrm{D}_{r \cdot g, \mathrm{M}}=\frac{1}{\sqrt{s}} \mathrm{D}_{s \cdot g, \mathrm{M}}
$$

as required.

We can now verify without difficulty that $(\mathscr{L}, \mathscr{D})$ is a full quantum isometry from $\Omega_{r}$ to $\Omega_{s}$, where

$$
\mathscr{L}=\operatorname{Id}_{A_{\Theta} \rightarrow A_{\Theta}} \quad \text { and } \quad \mathscr{D}=\sqrt{\frac{r}{s}} \operatorname{Id}_{\chi_{\Theta} \rightarrow \chi_{\Theta}} .
$$

However, in order to give the reader an appreciation for the sophistication involved when trying to resolve more difficult propinquity problems (see the conclusion below), we will prove Theorem 5.1 directly. 
Proof of Theorem 5.1. By Lemma 5.10, we may define a bijection $\beta_{r, s}: \mathrm{D}_{r \cdot g, \mathrm{M}}^{1} \rightarrow \mathrm{D}_{s \cdot g, \mathrm{M}}^{1}$ by $\forall X \in \mathrm{D}_{r \cdot g, \mathrm{M}}^{1}: \quad \beta_{r, s}(X) \stackrel{\mathrm{df}}{=} \sqrt{\frac{r}{s}} \cdot X$.

It follows that

$$
\gamma_{r, s} \stackrel{\mathrm{df}}{=}\left(\Omega_{r}, \Omega_{s}, A_{\Theta}, 1_{A_{\Theta}}, \operatorname{Id}_{A_{\Theta} \rightarrow A_{\Theta}}, \operatorname{Id}_{A_{\Theta} \rightarrow A_{\Theta}}, \mathrm{D}_{r \cdot g, \mathrm{M}}^{1}, \operatorname{Id}_{\mathrm{D}_{r \cdot g, \mathrm{M}}^{1} \rightarrow \mathrm{D}_{r \cdot g, \mathrm{M}}^{1}}, \beta_{r, s}\right)
$$

is an $(F, G, H)$-modular bridge, whose associated numerical quantities we now seek to compute.

To perform these computations, first note that $\mathrm{bn}_{\gamma_{r, s}}$ is a seminorm on $A_{\Theta} \oplus A_{\Theta}$ such that

$$
\begin{aligned}
\forall a_{1}, a_{2} \in A_{\Theta}: \quad \operatorname{bn}_{\gamma_{r, s}}\left(a_{1}, a_{2}\right) & =\left\|\operatorname{Id}_{A_{\Theta} \rightarrow A_{\Theta}}\left(a_{1}\right) 1_{A_{\Theta}}-1_{A_{\Theta}} \operatorname{Id}_{A_{\Theta} \rightarrow A_{\Theta}}\left(a_{2}\right)\right\|_{A_{\Theta}} \\
& =\left\|a_{1}-a_{2}\right\|_{A_{\Theta}} .
\end{aligned}
$$

We can then make the following observations regarding the basic reach, height and imprint of $\gamma_{r, s}$ :

- The basic reach of $\gamma_{r, s}$ is 0 :

$$
\begin{aligned}
\rho_{b}\left(\gamma_{r, s}\right) & =\max \left(\sup _{\substack{a_{1} \in \operatorname{Dom}(\mathrm{L}) \\
\mathrm{L}\left(a_{1}\right) \leq 1}} \inf _{\substack{a_{2} \in \operatorname{Dom}(\mathrm{L}) \\
\mathrm{L}\left(a_{2}\right) \leq 1}}\left\|a_{1}-a_{2}\right\|_{A_{\Theta}}, \sup _{\substack{a_{2} \in \operatorname{Dom}(\mathrm{L}) \\
\mathrm{L}\left(a_{2}\right) \leq 1}} \inf _{\substack{a_{1} \in \operatorname{Dom}(\mathrm{L}) \\
\mathrm{L}\left(a_{1}\right) \leq 1}}\left\|a_{1}-a_{2}\right\|_{A_{\Theta}}\right) \\
& =\max \left(\sup _{\substack{\sup _{1} \in \operatorname{Dom}(\mathrm{L}) \\
\mathrm{L}\left(a_{1}\right) \leq 1}} 0, \sup _{\substack{a_{2} \in \operatorname{Dom}(\mathrm{L}) \\
\mathrm{L}\left(a_{2}\right) \leq 1}} 0\right)=\max (0,0)=0 .
\end{aligned}
$$

- The height of $\gamma_{r, s}$ is 0 : As $\mathscr{S}\left(A_{\Theta} \mid 1_{A_{\Theta}}\right)=\mathscr{S}\left(A_{\Theta}\right)$, we have

$$
\begin{aligned}
\varsigma\left(\gamma_{r, s}\right) & =\max \left(\begin{array}{l}
\operatorname{Haus}_{\mathrm{mk}_{\mathrm{L}}}\left(\mathscr{S}\left(A_{\Theta}\right),\left\{\phi \circ \mathrm{Id}_{A_{\Theta} \rightarrow A_{\Theta}} \mid \phi \in \mathscr{S}\left(A_{\Theta}\right)\right\}\right) \\
\operatorname{Haus}_{\mathrm{mk}_{\mathrm{L}}}\left(\mathscr{S}\left(A_{\Theta}\right),\left\{\psi \circ \mathrm{Id}_{A_{\Theta} \rightarrow A_{\Theta}} \mid \psi \in \mathscr{S}\left(A_{\Theta}\right)\right\}\right)
\end{array}\right) \\
& =\max \left(\operatorname{Haus}_{\mathrm{mk}_{\mathrm{L}}}\left(\mathscr{S}\left(A_{\Theta}\right), \mathscr{S}\left(A_{\Theta}\right)\right), \operatorname{Haus}_{\mathrm{mk}_{\mathrm{L}}}\left(\mathscr{S}\left(A_{\Theta}\right), \mathscr{S}\left(A_{\Theta}\right)\right)\right) \\
& =\max (0,0)=0 .
\end{aligned}
$$

- The imprint of $\gamma_{r, s}$ is 0 :

$$
\begin{aligned}
\varpi\left(\gamma_{r, s}\right)= & \max \left(\operatorname{Haus}_{\mathrm{k}_{\Omega_{r}}}\left(\operatorname{Range}\left(\operatorname{Id}_{\mathrm{D}_{r \cdot g, \mathrm{M}}^{1} \rightarrow \mathrm{D}_{r \cdot g, \mathrm{M}}^{1}}\right), \mathrm{D}_{r \cdot g, \mathrm{M}}^{1}\right),\right. \\
& \left.\operatorname{Haus}_{\mathrm{k}_{\Omega_{s}}}\left(\operatorname{Range}\left(\beta_{r, s}\right), \mathrm{D}_{s \cdot g, \mathrm{M}}^{1}\right)\right) \\
= & \max \left(\operatorname{Haus}_{\mathrm{k}_{\Omega_{r}}}\left(\mathrm{D}_{r \cdot g, \mathrm{M}}^{1}, \mathrm{D}_{r \cdot g, \mathrm{M}}^{1}\right), \operatorname{Haus}_{\mathrm{k}_{\Omega_{s}}}\left(\mathrm{D}_{s \cdot g, \mathrm{M}}^{1}, \mathrm{D}_{s \cdot g, \mathrm{M}}^{1}\right)\right) \\
= & \max (0,0)=0 .
\end{aligned}
$$

Hence, $\lambda\left(\gamma_{r, s}\right)=\rho^{\sharp}\left(\gamma_{r, s}\right)$, i.e., the length of $\gamma_{r, s}$ equals its modular reach, which we will now prove is also 0 .

Observe for all $X, Y \in \mathrm{D}_{r \cdot g, \mathrm{M}}^{1}$ that

$$
\begin{aligned}
\operatorname{bn}_{\gamma_{r, s}}\left(\langle X \mid Y\rangle_{r \cdot g},\left\langle\beta_{r, s}(X) \mid \beta_{r, s}(Y)\right\rangle_{s \cdot g}\right) & =\left\|\langle X \mid Y\rangle_{r \cdot g}-\left\langle\beta_{r, s}(X) \mid \beta_{r, s}(Y)\right\rangle_{s \cdot g}\right\|_{A_{\Theta}} \\
& =\left\|\langle X \mid Y\rangle_{r \cdot g}-\left\langle\sqrt{\frac{r}{s}} \cdot X \mid \sqrt{\frac{r}{s}} \cdot Y\right\rangle_{s \cdot g}\right\|_{A_{\Theta}} \\
& =\left\|\langle X \mid Y\rangle_{r \cdot g}-\frac{r}{s} \cdot\langle X \mid Y\rangle_{s \cdot g}\right\|_{A_{\Theta}} \|_{A_{\Theta}} \\
& =\left\|r \cdot\langle X \mid Y\rangle_{g}-\frac{r}{s} \cdot\left(s \cdot\langle X \mid Y\rangle_{g}\right)\right\|_{A^{\prime}} \\
& =\left\|r \cdot\langle X \mid Y\rangle_{g}-r \cdot\langle X \mid Y\rangle_{g}\right\|_{A_{\Theta}}=0 .
\end{aligned}
$$


As the argument is symmetric in $X$ and $Y$, we also have for all $X, Y \in \mathrm{D}_{r \cdot g, \mathrm{M}}^{1}$ that

$$
\operatorname{bn}_{\gamma_{r, s}}\left(\langle Y \mid X\rangle_{r \cdot g},\left\langle\beta_{r, s}(Y) \mid \beta_{r, s}(X)\right\rangle_{s \cdot g}\right)=0
$$

SO

$$
\begin{aligned}
\operatorname{dn}_{\gamma_{r, s}} & \left(\operatorname{Id}_{\mathrm{D}_{r \cdot g, \mathrm{M}}^{1} \rightarrow \mathrm{D}_{r \cdot g, \mathrm{M}}^{1}}(X), \beta_{r, s}(X)\right)=\mathrm{dn}_{\gamma_{r, s}}\left(X, \beta_{r, s}(X)\right) \\
& =\sup \left(\left\{\begin{array}{l}
\left.\left.\left.\mathrm{bn}_{\gamma_{r, s}}\left(\left\langle X \mid \mathrm{Id}_{\mathrm{D}_{r \cdot g, \mathrm{M}}^{1} \rightarrow \mathrm{D}_{r \cdot g, \mathrm{M}}^{1}}(Y)\right\rangle_{r \cdot g},\left\langle\beta_{r, s}(X) \mid \beta_{r, s}(Y)\right\rangle_{s \cdot g}\right) \mid X \in \mathrm{D}_{r \cdot g, \mathrm{M}}^{1}\right\}\right)\right) \\
\mathrm{bn}_{\gamma_{r, s}}^{1}\left(\left\langle\operatorname{Id}_{\mathrm{D}_{r \cdot g, \mathrm{M}}^{1} \rightarrow \mathrm{D}_{r \cdot g, \mathrm{M}}^{1}}(Y) \mid X\right\rangle_{r \cdot g},\left\langle\beta_{r, s}(Y) \mid \beta_{r, s}(X)\right\rangle_{s \cdot g}\right)
\end{array}\right)\right. \\
& \left.=\sup \left(\left\{\begin{array}{l}
\mathrm{bn}_{\gamma_{r, s}}\left(\langle X \mid Y\rangle_{r \cdot g},\left\langle\beta_{r, s}(X) \mid \beta_{r, s}(Y)\right\rangle_{s \cdot g}\right) \\
\mathrm{bn}_{\gamma_{r, s}}\left(\langle Y \mid X\rangle_{r \cdot g},\left\langle\beta_{r, s}(Y) \mid \beta_{r, s}(X)\right\rangle_{s \cdot g}\right)
\end{array}\right) Y \in \mathrm{D}_{r \cdot g, \mathrm{M}}^{1}\right\}\right)=0
\end{aligned}
$$

for all $X \in \mathrm{D}_{r \cdot g, \mathrm{M}}^{1}$. Therefore,

$$
\begin{aligned}
\Lambda_{F, G, H}^{\bmod }\left(\Omega_{r}, \Omega_{s}\right) & \leq \lambda\left(\gamma_{r, s}\right)=\rho^{\sharp}\left(\gamma_{r, s}\right) \\
& =\sup \left(\left\{\operatorname{dn}_{\gamma_{r, s}}\left(\operatorname{Id}_{\mathrm{D}_{r \cdot g, \mathrm{M}}^{1} \rightarrow \mathrm{D}_{r \cdot g, \mathrm{M}}^{1}}(X), \beta_{r, s}(X)\right) \mid X \in \mathrm{D}_{r \cdot g, \mathrm{M}}^{1}\right\}\right)=0 .
\end{aligned}
$$

This completes the proof.

\section{Conclusion}

The only property of Levi-Civita connections used in this paper is metric compatibility. We could have allowed our D norms to depend not only on the choice of a Riemannian metric, but also on the choice of a connection that is not necessarily torsion-free. However, this would introduce an extra degree of variability that could unnecessarily complicate the study of convergence questions in modular propinquity.

Many unanswered questions remain. The four most important ones (in our opinion) are the following:

- Is $\mathrm{D}_{g}$ lower-semicontinuous on $\chi_{\Theta}^{\infty}$ for any Riemannian metric $g$ ? If this is the case, then (4) of Lemma 4.13 says that $\mathrm{D}_{g, \mathrm{M}}$ extends $\mathrm{D}_{g}$, which would render $\mathrm{D}_{g, \mathrm{M}}$ more manageable to deal with.

- Can we find a topology on $M_{n}\left(A_{\Theta}\right)$ so that if $h \rightarrow g$ in the space of Riemannian metrics with respect to this topology, then

$$
\Lambda_{F, G, H}^{\bmod }\left(\left(\chi_{\Theta},\langle\cdot \mid \cdot\rangle_{g}, \mathrm{D}_{g, \mathrm{M}}, A_{\Theta}, \mathrm{L}\right),\left(\chi_{\Theta},\langle\cdot \mid \cdot\rangle_{h}, \mathrm{D}_{h, \mathrm{M}}, A_{\Theta}, \mathrm{L}\right)\right) \rightarrow 0 ?
$$

This question can be posed so straightforwardly because we consider only Levi-Civita connections.

- Can we incorporate the Riemannian curvature operator [14, Definition 3.1] into the current work?

- Although Rosenberg considers only free modules in [14], can we extend our results to nonfree modules? In [7], Latrémolière establishes a technical propinquity result for metrized quantum vector bundles whose underlying modules are non-free Heisenberg modules over quantum 2-tori, but it is not clear at this time how his result may be extended to non-free Hilbert modules over quantum tori of arbitrary dimension. 


\section{Acknowledgments}

The author wishes to thank Konrad Aguilar and Frédéric Latrémolière for their generous help on the Gromov-Hausdorff propinquity. The author also wishes to thank Albert Sheu for his help in understanding Rosenberg's work on Levi-Civita connections. The warmest gratitude is reserved for the referees who offered valuable advice on how to significantly improve the quality of this paper.

\section{References}

[1] Aguilar K., Latrémolière F., Quantum ultrametrics on AF algebras and the Gromov-Hausdorff propinquity, Studia Math. 231 (2015), 149-193, arXiv:1511.07114.

[2] Bratteli O., Elliott G.A., Jorgensen P.E.T., Decomposition of unbounded derivations into invariant and approximately inner parts, J. Reine Angew. Math. 346 (1984), 166-193.

[3] Latrémolière F., Curved noncommutative tori as Leibniz quantum compact metric spaces, J. Math. Phys. 56 (2015), 123503, 16 pages, arXiv:1507.08771.

[4] Latrémolière F., The quantum Gromov-Hausdorff propinquity, Trans. Amer. Math. Soc. 368 (2016), 365411, arXiv:1302.4058.

[5] Latrémolière F., Quantum metric spaces and the Gromov-Hausdorff propinquity, in Noncommutative Geometry and Optimal Transport, Contemp. Math., Vol. 676, Amer. Math. Soc., Providence, RI, 2016, 47-133, arXiv:1506.04341.

[6] Latrémolière F., The modular Gromov-Hausdorff propinquity, arXiv:1608.04881.

[7] Latrémolière F., Convergence of the Heisenberg modules over quantum two-tori for the modular GromovHausdorff propinquity, arXiv:1703.07073.

[8] Latrémolière F., Packer J., Noncommutative solenoids and the Gromov-Hausdorff propinquity, Proc. Amer. Math. Soc. 145 (2017), 2043-2057, arXiv:1601.02707.

[9] Morrison T.J., Functional analysis: an introduction to Banach space theory, Pure and Applied Mathematics (New York), Wiley-Interscience, New York, 2001.

[10] Rieffel M.A., Metrics on states from actions of compact groups, Doc. Math. 3 (1998), 215-229, math.OA/9807084.

[11] Rieffel M.A., Metrics on state spaces, Doc. Math. 4 (1999), 559-600, math.OA/9906151.

[12] Rieffel M.A., Gromov-Hausdorff distance for quantum metric spaces, Mem. Amer. Math. Soc. 168 (2004), 1-65, math.OA/0011063.

[13] Rieffel M.A., Leibniz seminorms for "matrix algebras converge to the sphere", in Quanta of Maths, Clay Math. Proc., Vol. 11, Amer. Math. Soc., Providence, RI, 2010, 543-578, arXiv:0707.3229.

[14] Rosenberg J., Levi-Civita's theorem for noncommutative tori, SIGMA 9 (2013), 071, 9 pages, arXiv:1307.3775. 